\title{
Geometry of the 2+1 Black Hole
}

\author{
Máximo Bañados ${ }^{1,2, *}$, Marc Henneaux ${ }^{1,3, \# \text {, }}$ \\ Claudio Teitelboim ${ }^{1,2,4, *}$ and Jorge Zanelli ${ }^{1,2, *}$ \\ ${ }^{1}$ Centro de Estudios Cientificos de Santiago Casilla 16443, Santiago 9, Chile \\ ${ }^{2}$ Facultad de Ciencias, Universidad de Chile, Casilla 653, Santiago, Chile. \\ 3 Faculté des Sciences, Université Libre de Bruxelles, Belgium. \\ 4 Institute for Advanced Study, Olden Lane, Princeton, New Jersey 08540, USA.
}

Submitted to Phys. Rev. D.

November 1992 


\begin{abstract}
The geometry of the spinning black holes of standard Einstein theory in $2+1$ dimensions, with a negative cosmological constant and without couplings to matter, is analyzed in detail. It is shown that the black hole arises from identifications of points of anti-de Sitter space by a discrete subgroup of $S O(2,2)$. The generic black hole is a smooth manifold in the metric sense. The surface $r=0$ is not a curvature singularity but, rather, a singularity in the causal structure. Continuing past it would introduce closed timelike lines. However, simple examples show the regularity of the metric at $r=0$ to be unstable: couplings to matter bring in a curvature singularity there. Kruskal coordinates and Penrose diagrams are exhibited. Special attention is given to the limiting cases of (i) the spinless hole of zero mass, which differs from antide Sitter space and plays the role of the vacuum, and (ii) the spinning hole of maximal angular momentum . A thorough classification of the elements of the Lie algebra of $S O(2,2)$ is given in an Appendix.
\end{abstract}

PACS numbers 04.20 Jb, 97-60. Lf. 


\section{Introduction}

The black hole is one of the most fascinating structures that has ever emerged out of the theory of gravitation. And yet, it would seem fair to say, we are far from fully understanding it. It is therefore fortunate that full-fledged black holes have been found to exist [1] in the transparent setting of $2+1$ standard Einstein gravity [2].

The purpose of this article is to study in detail the geometry of the $2+1$ black hole without electric charge [3]. These results on the black hole geometry were only announced and briefly summarized in [1].

The plan of the article is the following: Section 2 deals with the action principle and its Hamiltonian version. The Hamiltonian is specialized to the case of axially symmetric time independent fields and the equations of motion are solved. The resulting metric has two integration constants which are next identified as the mass and angular momentum. This identification is achieved through an analysis of the surface integrals at spacelike infinity that must be added to the Hamiltonian in order to make it well defined. It is then shown that for a certain range of values of the mass and angular momentum the solution is a black hole. This black hole is shown to be quite similar to its $3+1$ counterpart -the Kerr solution. It has an ergosphere and an upper bound in angular momentum for any given mass.

The discussion of section 2 focuses on the physical properties of the black hole and ignores a question that must have been needling the geometer hiding within every theorist. The spacetime geometry of the black hole is one of constant negative curvature and therefore it is, locally, that of anti-de Sitter space. Thus, the black hole can only differ from anti-de Sitter space in its global properties. More precisely, as we shall see, the black hole arises from anti-de Sitter space through identifications of points of the latter by means of a discrete subgroup of its symmetry group 4 . Section 3 is devoted to this issue. The identifications are explicitly given and are, in particular, used to show that the black hole singularity at $r=0$ is not one in the metric, which is regular there, but rather a singularity in the causal structure. Continuing past $r=0$ would bring in closed timelike lines. When there is no angular momentum an additional pathology appears at $r=0$, a singularity in the manifold structure of the type present in the Taub-NUT space. This is dealt with in Appendix B. 
Once the identifications are geometrically understood, we pass, in Section 4, to exhibit special coordinate systems which reveal the causal structure. In particular, Kruskal coordinates are defined and the Penrose diagrams are drawn. Special issues pertaining to the extreme rotating black hole with non-zero mass and to the zero mass limit of a non-rotating hole ( "vacuum") are analyzed. Section 5 is devoted to some concluding remarks, showing the instability of the regularity of the metric at $r^{2}=0$ in the presence of matter. It is also briefly discussed how "chronology is protected" in the $2+1$ black hole.

The classification of the elements of the Lie algebra of the symmetry group $S O(2,2)$ is given in Appendix A.

\section{Action Principle, Equations of Motion and their Solutions.}

\subsection{Action Principle}

The action in lagrangian form may be taken to be

$$
I=\frac{1}{2 \pi} \int \sqrt{-g}\left[R+2 l^{-2}\right] d^{2} x d t+B^{\prime},
$$

where $B^{\prime}$ is a surface term and the radius $l$ is related to the cosmological constant by $-\Lambda=l^{-2}$. [ Note that, for convenience in what follows, the numerical factor $(16 \pi G)^{-1}$ in front of the action is taken to be $(2 \pi)^{-1}$, i.e., we set the gravitational constant $G$, which has the dimensions of an inverse energy, equal to $\left.\frac{1}{8}\right]$.

Extremization of the action with respect to the spacetime metric $g_{\mu \nu}(x, t)$, yields the Einstein field equations

$$
R_{\mu \nu}-\frac{1}{2} g_{\mu \nu}\left(R+2 l^{-2}\right)=0
$$

which, in a three dimensional spacetime, determine the full Riemann tensor as

$$
R_{\mu \nu \lambda \rho}=-l^{-2}\left(g_{\mu \lambda} g_{\nu \rho}-g_{\nu \lambda} g_{\mu \rho}\right)
$$

describing a symmetric space of constant negative curvature.

One may pass to the hamiltonian form of (2.1), which reads 


$$
I=\int\left[\pi^{i j} \dot{g}_{i j}-N^{\perp} \mathcal{H}_{\perp}-N^{i} \mathcal{H}_{i}\right] d^{2} x d t+B
$$

The surface term $B$ will be discussed below. It differs from the $B^{\prime}$ appearing in the lagrangian form because the corresponding volume integrals differ by a surface term. The surface deformation generators $\mathcal{H}_{\perp}, \mathcal{H}_{i}$ are given by

$$
\begin{aligned}
\mathcal{H}_{\perp} & =2 \pi g^{-1 / 2}\left(\pi^{i j} \pi_{i j}-\left(\pi_{i}^{i}\right)^{2}\right)-(2 \pi)^{-1} g^{1 / 2}\left(R+2 / l^{2}\right) \\
\mathcal{H}_{i} & =-2 \pi_{i / j}^{j}
\end{aligned}
$$

Extremizing the hamiltonian action with respect to the the lapse and shift functions $N^{\perp}, N^{i}$, yields the constraint equations $\mathcal{H}_{\perp}=0$ and $\mathcal{H}_{i}=0$ which are the $\perp, \perp$ and $\perp, i$ components of (2.2). Extremization with respect to the spatial metric $g_{i j}$ and its conjugate momentum $\pi^{i j}$, yields the purely spatial part of the second order field equations (2.2), rewritten as a hamiltonian system of first order in time.

\subsection{Axially symmetric stationary field}

One may restrict the action principle to a class of fields that possess a rotational Killing vector $\partial / \partial \phi$ and a timelike Killing vector $\partial / \partial t$. If the radial coordinate is properly adjusted, the line element may be written as

$$
\begin{aligned}
d s^{2}= & -\left(N^{\perp}\right)^{2}(r) d t^{2}+f^{-2}(r) d r^{2}+r^{2}\left(N^{\phi}(r) d t+d \phi\right)^{2} \\
& 0 \leq \phi<2 \pi, \quad t_{1} \leq t \leq t_{2}
\end{aligned}
$$

The form of the momenta $\pi^{i j}$ may be obtained from (2.7) through their relation $\pi^{i j}=-(1 / 2 \pi) g^{-1 / 2}\left(K^{i j}-K g^{i j}\right)$ with the extrinsic curvature $K_{i j}$, which, for a timeindependent metric, simply reads $2 N^{\perp} K_{i j}=\left(N_{i \mid j}+N_{j \mid i}\right)$. This gives as the only component of the momentum,

$$
\pi_{\phi}^{r}=\frac{l}{2 \pi} p(r)
$$

If expressions (2.7), (2.8) are introduced in the action, one finds

$$
I=-\left(t_{2}-t_{1}\right) \int d r\left[N(r) \mathcal{H}(r)+N^{\phi} \mathcal{H}_{\phi}\right]+B
$$


with

$$
\begin{aligned}
\mathcal{H} & \equiv 2 \pi f(r) \mathcal{H}_{\perp}=2 l^{2} \frac{p^{2}}{r^{3}}+\left(f^{2}\right)^{\prime}-2 \frac{r}{l^{2}} \\
\mathcal{H}_{\phi} & =-2 l p^{\prime} \\
N(r) & =f^{-1} N^{\perp}
\end{aligned}
$$

\subsection{Solutions}

To find solutions under the assumptions of time independence and axial symmetry, one must extremize the reduced action (2.9). Variation with respect to $N$ and $N^{\phi}$, yields that the generators $\mathcal{H}$ and $\mathcal{H}_{\phi}$ must vanish. These constraint equations are readily solved to give

$$
\begin{aligned}
p & =-\frac{J}{2 l} \\
f^{2} & =-M+\left(\frac{r}{l}\right)^{2}+\frac{J^{2}}{4 r^{2}}
\end{aligned}
$$

where $M$ and $J$ are two constants of integration, which will be identified below as the mass and angular momentum, respectively.

Variation of the action with respect to $f^{2}$ and $p$ yields the equations

$$
\begin{aligned}
N^{\prime} & =0 \\
\left(N^{\phi}\right)^{\prime}+\frac{2 l p}{r^{3}} N & =0
\end{aligned}
$$

which determine $N$ and $N^{\phi}$ as

$$
\begin{aligned}
N & =N(\infty) \\
N^{\phi} & =-\frac{J}{2 r^{2}} N(\infty)+N^{\phi}(\infty)
\end{aligned}
$$

The constants of integration $N(\infty)$ and $N^{\phi}(\infty)$ are part of the specification of the coordinate system, which is not fully fixed by the form of the line element (2.7) (see below). 


\subsection{Surface integrals at infinity}

\subsubsection{Quick analysis}

We will be interested in including in the variational principle the class of fields that approach our solution (2.13), (2.15) at spacelike infinity. This means that the action should have an extremum under variations of $g_{i j}$ and $\pi^{i j}$ that for large $r$ approach the variations of the expressions (2.13), for any $\delta M$ and $\delta J$ and for fixed $N(\infty)$, $N^{\phi}(\infty)$. However, as seen most evidently from the reduced form (2.9) of the action, upon varying $g_{i j}$ and $\pi^{i j}$ one picks up a surface term. That is, one finds

$$
\begin{aligned}
\delta I= & \left(t_{2}-t_{1}\right)\left[N(\infty) \delta M-N^{\phi}(\infty) \delta J\right]+\delta B \\
& +(\text { Terms vanishing when the equations of motion hold })
\end{aligned}
$$

Now, one must demand that when the equations of motion hold, the variation of the action should be zero[5]. Therefore, the boundary term $B$ in the action must be adjusted so as to cancel the first two terms on the right side of (2.16). Thus, we put

$$
B=\left(t_{2}-t_{1}\right)\left(-N(\infty) M+N^{\phi}(\infty) J\right)
$$

Equation (2.17) identifies $M$ as the mass and $J$ as the angular momentum. This is because they appear as conjugates to the asymptotic displacements $N(\infty)$ and $N^{\phi}(\infty)$. (The minus sign in front of $N(\infty)$ appears because, conventionally, one introduces a minus sign in the generator when the displacement is along a timelike direction.) That $N^{\phi}$ is the angular displacement is evident. However, the fact that the rescaled lapse $N$ given by (2.12) appears in (2.17) rather than the original $N^{\perp}$, deserves explanation. The reason is the following. The normal component of the deformation that joins the surface of time $t$ and that of time $t+\delta t$ is $\delta \xi=\mathbf{n} N^{\perp} \delta t$, where $\mathbf{n}$ is the unit normal. But the unit normal does not approach a Killing vector at infinity. If one multiplies it by $f$, one obtains, at infinity, a Killing vector $\mathbf{K}=\mathbf{n} f$ whose norm $\mathbf{K} \cdot \mathbf{K}=-f^{2}$ is independent of $N(\infty)$. The displacement $N(\infty) \delta t$ ("Killing time") is the component of the deformation $\delta \xi$ along $\mathbf{K}$. 


\subsubsection{Detailed analysis}

The preceding argument gives a quick way of obtaining the surface integrals that must be added to the action. It also puts in evidence the physical meaning of $M$ and $J$. However, a more careful analysis is needed. One knows that in a gauge theory such as General Relativity the conserved quantities are related to the asymptotic symmetry group. This fact already emerged in the previous discussion where "displacements at infinity" played the key role. For $2+1$ spacetime dimensions with a negative cosmological constant, this asymptotic group is infinite dimensional and contains $S O(2,2)$ as a subgroup. The asymptotic Killing vectors $\partial / \partial \phi$ and $\mathbf{K}=N(\infty)^{-1} \partial_{t}$ that appeared above are two of the generators in the Lie algebra of $S O(2,2)$. Thus, what we have called "Killing time displacements" are not "translations" but -rather- $S O(2,2)$ boosts.

The general analysis of the asymptotic symmetry group of $2+1$ gravity has been given in [6]. We briefly recall here its key aspects and apply them to the present treatment.

One considers all metrics that for large $r$ become

$$
d s^{2} \longrightarrow-\left(\frac{r}{l}\right)^{2} d t^{2}+\left(\frac{r}{l}\right)^{2} d r^{2}+r^{2} d \phi^{2},
$$

[There is no loss of generality -in this context- in taking $N(\infty)=1$ and $N^{\phi}(\infty)=0$. One must only remember that for any given spacetime the surface integrals are to be calculated in a coordinate system obeying these conditions.]

The precise way in which $d s^{2}$ approaches (2.18) for large $r$ is obtained by acting on the solution (2.13), (2.15), with all possible anti-de Sitter group transformations. The rationale for this procedure is that one wants to have at least $S O(2,2)$ as an asymptotic symmetry group. This is because the metric (2.18) coincides with the asymptotic form of the anti-de Sitter metric, which has $S O(2,2)$ as its (exact) symmetry group. The remarkable feature is that the resulting class of allowed asymptotic metrics admits a much larger symmetry group.

The asymptotic symmetry group turns out to be the conformal group. The conformal group may be defined as the group of all transformations that leave invariant the cylinder at infinity, up to a Weyl rescaling. The conformal Killing vectors obey 


$$
\xi_{\alpha ; \beta}+\xi_{\beta ; \alpha}-\frac{1}{2} g_{\alpha \beta} \xi_{; \lambda}^{\lambda}=0
$$

The Lie algebra of the conformal group consists of two copies of the Virasoro algebra. Therefore the conserved charges of $2+1$ gravity are two sets $L_{n}, K_{n}$ of Virasoro generators $(n=0, \pm 1, \pm 2, \ldots)$. Of these, the six $S O(2,2)$ generators are $L_{0}, L_{1}, L_{-1}, K_{0}, K_{1}, K_{-1}$, which form a subalgebra .

The $L_{n}$ and $K_{n}$ obey the Virasoro algebra with a central charge proportional to the radius of curvature. One has, in terms of non-quantum Poisson brackets,

$$
\begin{aligned}
{\left[L_{n}, L_{m}\right] } & =-i\left\{(n-m) L_{n+m}+l \cdot n\left(n^{2}-1\right) \delta_{n,-m}\right\} \\
{\left[K_{n}, K_{m}\right] } & =-i\left\{(n-m) K_{n+m}+l \cdot n\left(n^{2}-1\right) \delta_{n,-m}\right\} \\
{\left[L_{n}, K_{m}\right] } & =0
\end{aligned}
$$

In the normalization for the central charge that has become standard in string theory, one has

$$
c=12 l / \hbar
$$

The metric given by (2.13), (2.15) has only two charges which are non-zero $\left(M=K_{0}+L_{0}, \quad J=K_{0}-L_{0}\right)$. However, by acting with the asymptotic group one can endow it with other charges, much as by boosting a Schwarzschild solution one may endow it with linear momentum.

\subsection{The Black Hole}

The lapse function $N^{\perp}$ vanishes for two values of $r$ given by

$$
r_{ \pm}=l\left[\frac{M}{2}\left(1 \pm \sqrt{1-\left(\frac{J}{M l}\right)^{2}}\right)\right]^{1 / 2} .
$$

whereas $g_{00}$ vanishes at

$$
r_{\text {erg }}=l M^{1 / 2}
$$

These three special values of $r$ obey 


$$
r_{-} \leq r_{+} \leq r_{\text {erg }}
$$

Just as it happens in $3+1$ dimensions for the Kerr metric, $r_{+}$is the black hole horizon, $r_{e r g}$ is the surface of infinite redshift and the region between $r_{+}$and $r_{e r g}$ is the ergosphere. In order for the solution to describe a black hole, one must have

$$
M>0, \quad|J| \leq M l .
$$

In the extreme case $|J|=M l$, both roots of $N^{2}=0$ coincide. Note that the radius of curvature $l=(-\Lambda)^{-1 / 2}$ provides the length scale necessary in order to have a horizon in a theory in which the mass is dimensionless. If one lets $l$ grow very large the black hole exterior is pushed away to infinity and one is left just with the inside.

The vacuum state, namely what is to be regarded as empty space, is obtained by making the black hole disappear. That is, by letting the horizon size go to zero. This amounts to letting $M \rightarrow 0$, which requires $J \rightarrow 0$ on account of (2.25). One thus obtains the line element

$$
d s_{v a c}^{2}=-(r / l)^{2} d t^{2}+(r / l)^{-2} d r^{2}+r^{2} d \phi^{2} .
$$

As $M$ grows negative one encounters the solutions studied previously in [7]. The conical singularity that they possess is naked, just as the curvature singularity of a negative mass black hole in $3+1$ dimensions. Thus, they must, in the present context, be excluded from the physical spectrum. There is however an important exceptional case. When one reaches $M=-1$ and $J=0$ the singularity disappears. There is no horizon, but there is no singularity to hide either. The configuration

$$
d s^{2}=-\left(1+(r / l)^{2}\right) d t^{2}+\left(1+(r / l)^{2}\right)^{-1} d r^{2}+r^{2} d \phi^{2}
$$

(anti-de Sitter space) is again permissible.

Therefore, one sees that anti-de Sitter space emerges as a "bound state", separated from the continuous black hole spectrum by a mass gap of one unit. This state cannot be deformed continuously into the vacuum (2.26), because the deformation would require going through a sequence of naked singularities which are not included in the configuration space. 
Note that the zero point of energy has been set so that the mass vanishes when the horizon size goes to zero. This is quite natural. It is what is done in $3+1$ dimensions. In the past, the zero of energy has been adjusted so that anti-de Sitter space has zero mass instead. Quite apart from this difference, the key point is that the black hole spectrum lies above the limiting case $M=0$.

We now pass, in the next section, to a detailed study of the geometry of the black hole.

\section{Black Hole as Anti-de Sitter Space Factored by a Subgroup of its Symmetry Group}

We will show in this section that the black hole arises from anti-de Sitter space through identifications by means of a discrete subgroup of its isometry group $S O(2,2)$. This implies that the black hole is a solution of the source-free Einstein equations everywhere, including $r=0$. As we shall also see, the type of "singularity" that is found at $r=0$ is -generically- one in the causal structure and not in the curvature, which is everywhere finite (and constant). It should be emphasized that this statement means that $r=0$ is not a conical singularity.

To proceed with the analysis we first review the properties of anti-de Sitter space.

\subsection{Anti-de Sitter Space in 2+1 Dimensions}

\subsubsection{Metric}

Anti-de Sitter space can be defined in terms of its embedding in a four dimensional flat space of signature $(--++)$

$$
d s^{2}=-d u^{2}-d v^{2}+d x^{2}+d y^{2}
$$

through the equation

$$
-v^{2}-u^{2}+x^{2}+y^{2}=-l^{2}
$$

A system of coordinates covering the whole of the manifold may be introduced by setting 


$$
u=l \cosh \mu \sin \lambda, \quad v=l \cosh \mu \cos \lambda
$$

with $l \sinh \mu=\sqrt{x^{2}+y^{2}}$ and $0 \leq \mu<\infty, 0 \leq \lambda<2 \pi$. Inserting (3.3) into (3.1) gives

$$
d s^{2}=l^{2}\left(-\cosh ^{2} \mu d \lambda^{2}+\frac{d x^{2}+d y^{2}}{l^{2}+x^{2}+y^{2}}\right)
$$

an expression that can be further simplified by passing to polar coordinates in the $x-y$ plane

$$
x=l \sinh \mu \cos \theta, \quad y=l \sinh \mu \cos \theta,
$$

which yields

$$
d s^{2}=l^{2}\left[-\cosh ^{2} \mu d \lambda^{2}+d \mu^{2}+\sinh ^{2} \mu d \theta^{2}\right]
$$

for the metric of anti-de Sitter space.

Because $\lambda$ is an angle, there are closed timelike curves in anti-de Sitter space (for instance $\left.\mu=\mu_{0}, \theta=\theta_{0}\right)$. For this reason, one "unwraps" the $\lambda$ coordinate, that is, one does not identify $\lambda$ with $\lambda+2 \pi$. The space thus obtained is the universal covering of anti-de Sitter space. It is this space which, by a common abuse of language, will be called anti-de Sitter space in the sequel. If the unwrapped $\lambda$ is denoted by $t / l$ and if one sets $r=l \sinh \mu$, one obtains

$$
d s^{2}=\left((r / l)^{2}+1\right) d t^{2}+\left((r / l)^{2}+1\right)^{-1} d r^{2}+r^{2} d \theta^{2}
$$

which is the metric (2.7) with $M=-1, J=0($ and $\phi$ replaced by $\theta)$.

\subsubsection{Isometries}

By construction, the anti-de Sitter metric is invariant under $S O(2,2)$. The Killing vectors are

$$
J_{a b}=x_{b} \frac{\partial}{\partial x^{a}}-x_{a} \frac{\partial}{\partial x^{b}}
$$

where $x^{a}=(v, u, x, y)$ or, in detail 


$$
\begin{array}{ll}
J_{01}=v \partial_{u}-u \partial_{v} & J_{02}=x \partial_{v}+v \partial_{x} \\
J_{03}=y \partial_{v}+v \partial_{y} & J_{12}=x \partial_{u}+u \partial_{x} \\
J_{13}=y \partial_{u}+u \partial_{y} & J_{23}=y \partial_{x}-x \partial_{y}
\end{array}
$$

The vector $J_{01}$ generates "time displacements" $\left(J_{01}=\partial_{\lambda}\right)$ whereas $J_{23}$ generates rotations in the $x-y$ plane $\left(J_{23}=\partial_{\theta}\right)$. The most general Killing vector is given by

$$
\frac{1}{2} \omega^{a b} J_{a b}, \quad \omega^{a b}=-\omega^{b a}
$$

and is thus determined by an antisymmetric tensor in $\mathbf{R}^{4}$.

\subsubsection{Poincaré Coordinates}

The coordinates defined by

$$
z=\frac{l}{u+x}, \quad \beta=\frac{y}{u+x}, \quad \gamma=\frac{-v}{u+x} .
$$

are called Poincaré coordinates. They only cover part of the space, namely just one of the infinitely many regions where $u+x$ has a definite sign (see Fig. 1). These coordinates are therefore not well adapted to the study of global properties. In terms of $(z, \beta, \gamma)$ the anti-de Sitter line element reads

$$
d s^{2}=l^{2}\left[\frac{d z^{2}+d \beta^{2}-d \gamma^{2}}{z^{2}}\right] .
$$

For $u+x>0$ one has $z>0$ and for $u+x<0$ one has $z<0$. One can also find analogous Poincaré coordinates for each of the regions where $u-x$ has a definite sign.

\section{$3.2 \quad$ Identifications}

\subsubsection{Identification subgroup associated with a Killing vector}

Any Killing vector $\xi$ defines a one parameter subgroup of isometries of anti-de Sitter space

$$
P \rightarrow e^{t \xi} P
$$


The mappings of (3.13) for which $t$ is an integer multiple of a basic "step", taken conventionally as $2 \pi$,

$$
P \rightarrow e^{t \xi} P, \quad t=0, \pm 2 \pi, \pm 4 \pi, \ldots
$$

define what we will call the identification subgroup

Since the transformations (3.14) are isometries, the quotient space obtained by identifying points that belong to a given orbit of the identification subgroup, inherits from anti-de Sitter space a well defined metric which has constant negative curvature. The quotient space thus remains a solution of the Einstein equations.

The identification process makes the curves joining two points of anti-de Sitter space that are on the same orbit to be closed in the quotient space. In order for the quotient space to have an admissible causal structure, these new closed curves should not be timelike or null. A necessary condition for the absence of closed timelike lines is that the Killing vector $\xi$ be spacelike,

$$
\xi \cdot \xi>0
$$

This condition is not sufficient in general. However, as it will be shown in Sec. 3.2.5, it turns out to be so for the particular Killing vectors employed in the identifications leading to the black hole.

\subsubsection{Singularities in the causal structure}

There are some Killing vectors that do fulfill (3.15) everywhere in anti-de Sitter space, for example $\frac{\partial}{\partial \theta}$, where $\theta$ is the angular coordinate appearing in (3.6).

However, the Killing vectors appearing in the identifications that give rise to the black hole are timelike or null in some regions. These regions must be cut out from anti-de Sitter space to make the identifications permissible. The resulting space -which we denote (adS)'- is invariant under (3.13) because the norm of a Killing vector is constant along its orbits. Hence, the quotient can still be taken.

The space (adS)' is geodesically incomplete since one can find geodesics that go from $\xi \cdot \xi>0$ to $\xi \cdot \xi<0$. From the point of view of $(\operatorname{adS})^{\prime}$-i.e., prior to the identifications- it is quite unnatural to remove the regions where $\xi \cdot \xi$ is not positive. However, once the identifications are made, the frontier of the region $\xi \cdot \xi>0$, i.e., 
the surface $\xi \cdot \xi=0$, appears as a singularity in the causal structure of spacetime, since continuing beyond it would produce closed timelike curves.

For this reason, the region $\xi \cdot \xi=0$ may be regarded as a true singularity in the quotient space. If this point of view is taken, -as it is done here- the only incomplete geodesics are those that hit the singularity, just as in the $3+1$ black hole. It should be stressed that the surface $\xi \cdot \xi=0$ is a singularity only in the causal structure. It is not a conical curvature singularity of the type discussed in [7]. Indeed, the quotient space is smooth [8]. Its curvature tensor is everywhere regular and given by

$$
R_{\mu \nu \lambda \rho}=-l^{-2}\left(g_{\mu \lambda} g_{\nu \rho}-g_{\nu \lambda} g_{\mu \rho}\right)
$$

The fundamental group of the quotient space is non trivial and isomorphic to the identification subgroup. The orbits of the Killing vectors define closed curves that cannot be continuously shrunk to a point. The "origin" $\xi \cdot \xi=0$ is neither a point nor a circle. It is a surface. The topology of $\xi \cdot \xi=0$, and also that of the whole quotient space, can be inferred by inspection of the Penrose diagram in Fig. 4c. One finds that the black hole is topologically $\mathbf{R}^{2} \times S^{1}$ and that the surface $\xi \cdot \xi=0$ has infinitely many connected pieces, each of which is a cylinder whose circular sections are null.

\subsubsection{Explicit form of the identifications}

We claim that the black hole solutions are obtained by making identifications of the type described above by the discrete group generated by the Killing vector

$$
\xi=\frac{r_{+}}{l} J_{12}-\frac{r_{-}}{l} J_{03}-J_{13}+J_{23}
$$

where the $J_{a b}$ are given by (3.8). The antisymmetric tensor $\omega^{a b}$ defined by (3.17) through $\xi=\frac{1}{2} \omega^{a b} J_{a b}$, is easily verified to possess real eigenvalues, namely, $\pm r_{+} / l$ and $\pm r_{-} / l$. The corresponding Casimir invariants $I_{1}=\omega_{a b} \omega^{a b}$ and $I_{2}=\frac{1}{2} \epsilon_{a b c d} \omega^{a b} \omega^{c d}$ are

$$
I_{1}=-\frac{2}{l^{2}}\left(r_{+}^{2}+r_{-}^{2}\right)=-2 M, \quad I_{2}=-\frac{4}{l^{2}} r_{+} r_{-}=-2 \frac{|J|}{l}
$$


According to the classification given in Appendix A the Killing vector (3.17) is of type $\mathbf{I}_{b}$ when $r_{+} \neq r_{-}$, of type $\mathbf{I I}_{a}$ when $r_{+}=r_{-} \neq 0$ and of type $\mathbf{I I I}^{+}$when $r_{+}=r_{-}=0$.

To prove that the identifications by $e^{2 \pi k \xi}$ yield the black hole metric, we start by considering the non - extreme case $r_{+}^{2}-r_{-}^{2}>0$. In that case, by performing an $S O(2,2)$ transformation, one can eliminate the last term in (3.17) and replace $\xi$ by the simpler expression

$$
\xi^{\prime}=\frac{r_{+}}{l} J_{12}-\frac{r_{-}}{l} J_{03}
$$

This follows from the analysis of Appendix A, where it is shown that any $\operatorname{SO}(2,2)$ element with unequal real eigenvalues can be brought into the form (3.19) by an $S O(2,2)$ transformation. Alternatively, one may rewrite (3.17) in Poincaré coordinates as

$$
-\xi=\frac{r_{+}}{l}\left(z \frac{\partial}{\partial z}+\beta \frac{\partial}{\partial \beta}+\gamma \frac{\partial}{\partial \gamma}\right)-\frac{r_{-}}{l}\left(\beta \frac{\partial}{\partial \gamma}+\gamma \frac{\partial}{\partial \beta}\right)+\frac{\partial}{\partial \beta}
$$

and observe that the shifts

$$
\begin{aligned}
& \beta \rightarrow \beta-\frac{r_{+}}{r_{+}^{2}-r_{-}^{2}} \\
& \gamma \rightarrow \gamma-\frac{r_{-}}{r_{+}^{2}-r_{-}^{2}}
\end{aligned}
$$

-which are $S O(2,2)$ isometries- eliminate $\frac{\partial}{\partial \beta}$ in $(3.20)$.

The norm of $\xi^{\prime}$ is given by

$$
\xi^{\prime} \cdot \xi^{\prime}=\frac{r_{+}^{2}}{l^{2}}\left(u^{2}-x^{2}\right)+\frac{r_{-}^{2}}{l^{2}}\left(v^{2}-y^{2}\right)
$$

or, using (3.2),

$$
\xi^{\prime} \cdot \xi^{\prime}=\frac{r_{+}^{2}-r_{-}^{2}}{l^{2}}\left(u^{2}-x^{2}\right)+r_{-}^{2}
$$

Accordingly, the allowed region where $\xi^{\prime} \cdot \xi^{\prime}>0$ is

$$
\frac{-r_{-}^{2} l^{2}}{r_{+}^{2}-r_{-}^{2}}<u^{2}-x^{2}<\infty
$$


The region $\xi^{\prime} \cdot \xi^{\prime}>0$ can be divided in an infinite number of regions of three different types bounded by the null surfaces $u^{2}-x^{2}=0$ or $v^{2}-y^{2}=l^{2}-\left(u^{2}-x^{2}\right)=0$. These regions are:

Regions of type I: Smallest connected regions with $u^{2}-x^{2}>l^{2}$ and $y$ and $u$ of definite sign. These regions have no intersection with $y=0$ since this would violate $u^{2}-x^{2}=l^{2}+y^{2}-v^{2}>l^{2}$. These regions are called "the outer regions". The norm of the Killing vector fulfills $r_{+}^{2}<\xi^{\prime} \cdot \xi^{\prime}<+\infty$.

Regions of type II: Smallest connected regions with $0<u^{2}-x^{2}<l^{2}$ and $u$ and $v$ of definite sign. These regions are called "the intermediate regions". The norm of the Killing vector fulfills $r_{-}^{2}<\xi^{\prime} \cdot \xi^{\prime}<r_{+}^{2}$.

Regions of type III: Smallest connected regions with $-\frac{r_{-}^{2} l^{2}}{r_{+}^{2}-r_{-}^{2}}<u^{2}-x^{2}<0$ and $x$ and $v$ of definite sign. These regions are called "the inner regions" and only exist for $r_{-} \neq 0$. They do not intersect the $x=0$ plane. The norm of the Killing vector fulfills $0<\xi^{\prime} \cdot \xi^{\prime}<r_{-}^{2}$

The frontiers between the various regions are lightlike surfaces (the horizons!). Each region of type $\mathbf{I}$ has one region of type II in its future and one in its past. For $r_{-} \neq 0$, two situations are found for each region of type II: (i) it has one region of type II and two regions of type $\mathbf{I}$ in its future as well as one region of type II and two regions of type III in its past, or conversely (ii) the same description with I and III interchanged. Finally, each region of type III has one region of type II in its future and another one in its past. This is shown in Figures (2.a,b,c). Let us now choose three contiguous regions of types I, II and III (one of each type). In these regions we introduce a $(t, r, \phi)$ - parametrization as follows (we assume for definiteness $u, y>0$ in $\mathbf{I}, u,-v>0$ in II and $x,-v>0$ in III).

Region I. $r_{+}<r$ :

$$
\begin{aligned}
u & =\sqrt{A(r)} \cosh \tilde{\phi}(t, \phi) \\
x & =\sqrt{A(r)} \sinh \tilde{\phi}(t, \phi) \\
y & =\sqrt{B(r)} \cosh \tilde{t}(t, \phi) \\
v & =\sqrt{B(r)} \sinh \tilde{t}(t, \phi)
\end{aligned}
$$

Region II. $r_{-}<r<r_{+}$:

$$
u=\sqrt{A(r)} \cosh \tilde{\phi}(t, \phi)
$$




$$
\begin{aligned}
x & =\sqrt{A(r)} \sinh \tilde{\phi}(t, \phi) \\
y & =-\sqrt{-B(r)} \sinh \tilde{t}(t, \phi) \\
v & =-\sqrt{-B(r)} \cosh \tilde{t}(t, \phi)
\end{aligned}
$$

Region III. $0<r<r_{-}$:

$$
\begin{aligned}
u & =\sqrt{-A(r)} \sinh \tilde{\phi}(t, \phi) \\
x & =\sqrt{-A(r)} \cosh \tilde{\phi}(t, \phi) \\
y & =-\sqrt{-B(r)} \sinh \tilde{t}(t, \phi) \\
v & =-\sqrt{-B(r)} \cosh \tilde{t}(t, \phi)
\end{aligned}
$$

In (3.26), (3.27) and (3.28) we have set

$$
\begin{aligned}
A(r) & =l^{2}\left(\frac{r^{2}-r_{-}^{2}}{r_{+}^{2}-r_{-}^{2}}\right) & & B(r)=l^{2}\left(\frac{r^{2}-r_{+}^{2}}{r_{+}^{2}-r_{-}^{2}}\right) \\
\tilde{t} & =(1 / l)\left(r_{+} t / l-r_{-} \phi\right) & & \tilde{\phi}=(1 / l)\left(-r_{-} t / l+r_{+} \phi\right)
\end{aligned}
$$

In the coordinates $t, r, \phi$, the metric becomes

$$
d s^{2}=-\left(N^{\perp}\right)^{2} d t^{2}+\left(N^{\perp}\right)^{-2} d r^{2}+r^{2}\left(N^{\phi} d t+d \phi\right)^{2}
$$

with $-\infty<t<\infty,-\infty<\phi<\infty$ i.e., it is the black hole metric but with $\phi$ a non-periodic coordinate. The Killing vector $\xi^{\prime}$ reads

$$
\xi^{\prime}=\frac{\partial}{\partial \phi}
$$

By making the identification

$$
\phi \rightarrow \phi+2 k \pi,
$$

one gets the black hole spacetime as claimed above.

It is clear from the construction that the coordinate system $t, r, \phi$ does not cover the domain $\xi^{\prime} \cdot \xi^{\prime}>0$ entirely, since it only covers one region of each type. If $r_{-}=0$ (in which case region III does not exist), this is only half of one connected component of the domain $\xi^{\prime} \cdot \xi^{\prime}>0$. If $r_{-} \neq 0$, each of the regions I, II and 
III should be repeated an infinite number of times to completely cover the domain $\xi^{\prime} \cdot \xi^{\prime}>0$ which is now connected. This infinite pattern follows from the fact that one is dealing with the universal covering space of anti-de Sitter space and this will reappear in the Penrose diagrams given below.

It is worthwhile emphasizing that it is the identification (3.32) that makes the black hole. If one does not say that $\phi$ is an angle, one simply has a portion of anti-de Sitter space and the horizon is just that of an accelerated observer [9].

\subsubsection{Extreme case}

The above derivation cannot be repeated in the extreme case $r_{+}=r_{-}$. This is because the Killing vector (3.17) is now of a different type than (3.19). According to the classification given in the Appendix, when $r_{+}=r_{-}$, (3.17) is of type $\mathbf{I I}_{a}$, while (3.19) is of type $\mathbf{I}_{b}$ with doubly degenerate roots. Hence, there is no $S O(2,2)$ transformation mapping one to the other.

One can nevertheless argue that the identifications for anti-de Sitter space generated by (3.17) yield the extreme black hole without exhibiting the precise coordinate transformation that brings $\xi$ into the form $\partial / \partial \phi$. The argument runs as follows. The metric (3.30) is regular even if one sets $r_{+}^{2}=r_{-}^{2}$. When $\phi$ is not identified, it describes a portion of anti-de Sitter space for any value of $r_{+}^{2}-r_{-}^{2}>0$, hence it does so also in the limit $r_{+}-r_{-} \rightarrow 0$. Similarly, $\partial / \partial \phi$ is a Killing vector for any value of $r_{-}$ and $r_{+}$. By continuity, its Casimir invariants remain equal to $I_{1}=-2\left(r_{+}^{2}+r_{-}^{2}\right) / l^{2}$ and $I_{2}=-4 r_{+} r_{-} / l^{2}$ in the limit $r_{+}-r_{-} \rightarrow 0$. Hence, in the extreme case the vector $\partial / \partial \phi$ remains type $\mathrm{I}_{b}$ (with coincident roots) or becomes type $\mathbf{I I}_{a}$, since these are the only two types compatible with the given $I_{1}, I_{2}$. It is the latter alternative that is realized. Indeed, type $\mathbf{I}_{b}$ may be excluded by noticing that the corresponding Killing vector has constant norm equal to $r_{+}^{2}$, whereas $\partial / \partial \phi$ has a space-dependent norm equal to $r^{2}$. Thus $\partial / \partial \phi$ must be of type $\mathrm{II}_{a}$ and, thus, equal to (3.17) [up to a possible $S O(2,2)$ transformation that leaves the metric invariant].

The preceding argument already establishes that the black hole is obtained from anti-de Sitter space by an identification. However, for completeness we exhibit a change of coordinates in terms of which the identification just makes a coordinate periodic. The required coordinate transformation can be explicitly given in Poincaré coordinates. We start with the case $M=r_{+}=r_{-}=0$ ("the vacuum"), which is the 
more illuminating one.

For $M=0$, the region $\xi \cdot \xi>0$ splits into disjoint regions which are just the Poincaré patches $u+x>0$ or $u+x<0$. Hence, to describe a connected domain where $\xi \cdot \xi>0$, one can just consider a single Poincaré patch. In Poincaré coordinates the Killing vector $\xi$ is $-\frac{\partial}{\partial \beta}$ and hence, the identifications

$$
\beta \rightarrow \beta+2 k \pi
$$

in (3.12) lead to the black hole metric with $M=0$ upon setting $z=1 / r, \beta=\phi$ and $\gamma=t$.

[Note that as depicted in Fig 1, the horizon-singularity $r=0$ are the null surfaces $u+x=0$ delimiting the Poincaré region. Because the Killing vector $\xi$ is again spacelike on the other side of $u+x=0$, one can continue the solution with zero mass through $r=0$ to negative values of $r$ without encountering closed timelike curves. By doing so one includes, however, the closed lightlike curves that lie on the null surface $u+x=0$, as well as some singularities in the manifold structure of the type discussed in Appendix B.]

The coordinate transformation bringing the anti-de Sitter metric to the extreme case with $M \neq 0$ (and non-periodic in $\phi$ ) is more complicated. One needs in that case more than one Poincaré patch to cover the black-hole spacetime. Actually an infinite number of sets of patches is necessary, with each set containing one patch of each of the four types $u+x>0, u+x<0, u-x>0, u-x<0$. We merely give here that transformation in one of the patches $u+x>0$, for $r>r_{+}$.

$$
\begin{aligned}
\beta & =\frac{1}{2}\left(\frac{T}{l}+\phi+e^{2 r_{+} \phi}-\frac{1}{2 r_{+}}\right) \\
\gamma & =\frac{1}{2}\left(\frac{T}{l}+\phi-e^{2 r_{+} \phi}+\frac{1}{2 r_{+}}\right) \\
z & =\left[\frac{1}{2 r_{+}}\left(r^{2}-r_{+}^{2}\right)\right]^{-1 / 2} e^{r_{+} \phi}
\end{aligned}
$$

where $T$ is given by

$$
T=2 t-\frac{l^{2} r_{+}}{r^{2}-r_{+}^{2}}
$$


and fulfills $d T=2 d t+\frac{2 r_{+} l^{2} r d r}{\left(r^{2}-r_{+}^{2}\right)^{2}}$. By substituting (3.34)-(3.36) in the Poincaré metric, one gets the extreme black hole metric (with $N^{\phi}$ adjusted so that $N^{\phi}\left(r_{+}\right)=0$ ).

\subsubsection{Absence of Closed Timelike Curves}

We now complete the argument that there are no closed causal curves in the black hole solution. That is, we show that there is no non-spacelike, future-directed, curve lying in the region $\xi \cdot \xi>0$ of anti-de Sitter space and joining a point and its image generated by $\exp 2 \pi \xi$.

Since the surfaces $r=r_{+}$and $r=r_{-}$are null, a causal curve which leaves any one of the regions of types I, II or III through $r=r_{+}$or $r=r_{-}$can never re-enter it. Furthermore, since the images of a point are all in the same region as that point, it is sufficient to consider each of these regions separately.

In each of the regions of types I, II or III, the anti-de Sitter metric takes the form

$$
d s^{2}=-\left(N^{\perp}(r)\right)^{2} d t^{2}+\left(N^{\perp}(r)\right)^{-2} d r^{2}+r^{2}\left(N^{\phi} d t+d \phi\right)^{2}
$$

where $\phi$ goes from $-\infty$ to $+\infty$. Consider a causal curve $t(\lambda) r(\lambda)$ and $\phi(\lambda)$, where the parametrization is such that the tangent vector $(d t / d \lambda, d r / d \lambda, d \phi / d \lambda)$ does not vanish for any value of $\lambda$. The causal property of the curve reads

$$
\left(N^{\perp}\right)^{2}\left(\frac{d t}{d \lambda}\right)^{2}-\left(N^{\perp}\right)^{-2}\left(\frac{d r}{d \lambda}\right)^{2}-r^{2}\left(N^{\phi} \frac{d t}{d \lambda}+\frac{d \phi}{d \lambda}\right)^{2} \leq 0 .
$$

In order to join the point $\left(t_{0}, r_{0}, \phi_{0}\right)$ and $\left(t_{0}, r_{0}, \phi_{0}+2 k \pi\right)$, the causal curve would have to be such that $d t / d \lambda=0$ for some value of $\lambda$, since $t$ comes back to its initial value. But then, if $\left(N^{\perp}\right)^{2}>0$ it follows from (3.39) that $d r / d \lambda=d \phi / d \lambda=0$, leading to a contradiction. Similarly, if $\left(N^{\perp}\right)^{2}<0$ (region II), the fact that $d r / d \lambda=$ 0 for some value of $\lambda$ implies $d t / d \lambda=d \phi / d \lambda=0$, and the required contradiction.

It should be observed that if one were to admit the region $\xi \cdot \xi \leq 0$ in the solution, one could leave and re-enter the regions of type III through the surface $\xi \cdot \xi=0$, which is timelike for $J \neq 0$. (This is not possible when $J=0$ because the surface $\xi \cdot \xi=0$ is then null.) One would find that there are also closed timelike curves passing through points in region III. The boundary between the region where there are no closed causal curves and the region in which there are is then the null surface 
$r=r_{-}$. From the point of view of an outside observer staying at $r>r_{+}$, the inclusion or non-inclusion of the region $\xi \cdot \xi \leq 0$ is irrelevant and cannot be probed since the surface $r=r_{+}$remains in all cases an event horizon.

\subsubsection{Black Hole has only two Killing vectors}

The black hole metric was obtained in Sec. 3.2.3 under the assumption of existence of two commuting Killing vectors $\partial / \partial t$ and $\partial / \partial \phi$. One may ask whether there are any other independent Killing vectors. The answer to this question is in the negative as we now proceed to show.

Before any identifications are made one has the six independent Killing vectors $J_{a b}$ of anti-de-Sitter space. However, after the identifications, not all the corresponding vector fields will remain single valued in the quotient space.

A necessary and sufficient condition for an adS vector field $\eta$ to induce a well defined vector field on the quotient space is that $\eta$ be invariant under the transformation of the identification subgroup,

$$
(\exp 2 \pi \xi)^{*} \eta=\eta
$$

For a Killing vector, this condition becomes

$$
(\exp 2 \pi \xi) \eta(\exp 2 \pi \xi)^{-1}=\eta
$$

i.e.

$$
[\exp 2 \pi \xi, \eta]=0
$$

where $\xi$ and $\eta$ are viewed as $s o(2,2)$ matrices.

Now, the matrix $\xi$ can be decomposed as

$$
\xi=s+n
$$

where (i) $s$ and $n$ commute, (ii) $s$ is semi-simple with real eigenvalues; and (iii) $n$ is nilpotent (see Appendix A). Accordingly, the semi-simple part of $(\exp 2 \pi \xi)$ is $\exp 2 \pi s$ and its nilpotent part is $(\exp 2 \pi s)[(\exp 2 \pi n)-1]$. Any matrix commuting with $(\exp 2 \pi \xi)$ must thus separately commute with $(\exp 2 \pi s)$ and $(\exp 2 \pi n)$ (the semi-simple and nilpotent parts of a matrix can be expressed polynomially in terms of that matrix). This implies both 


$$
[s, \eta]=0
$$

(because the eigenvalues of the matrix $\exp 2 \pi s$ are real and positive, any matrix commuting with it must also commute with $\log (\exp 2 \pi s)=2 \pi s)$ and

$$
[n, \eta]=0
$$

(the nilpotent matrix $n$ can be expressed polynomially in terms of the nilpotent matrix $[(\exp 2 \pi n)-1]$ and must thus commute with $\eta)$. It follows from (3.44) and (3.45) that $\xi$ and $\eta$ commute,

$$
[\xi, \eta]=0 .
$$

The problem of finding all the Killing vectors of the black hole solution is thus equivalent to that of finding all the elements of the Lie algebra $s o(2,2)$ that commute with $\xi$.

In order to solve equation (3.46) for $\eta$, we observe that $s o(2,2)=s o(2,1) \oplus$ so $(2,1)$ and decompose accordingly $\xi$ into its self-dual and anti-self-dual parts,

$$
\xi=\xi^{+}+\xi^{-}
$$

Similarly,

$$
\eta=\eta^{+}+\eta^{-}
$$

The Equation (3.46) is equivalent to

$$
\left[\xi^{+}, \eta^{+}\right]=0, \quad\left[\xi^{-}, \eta^{-}\right]=0
$$

because self-dual and anti-self-dual elements automatically commute. Now, the only elements of $s o(2,1)$ that commute with a given non-zero element of $s o(2,1)$ are the multiples of that element. Therefore, since $\xi^{+}$and $\xi^{-}$are both non-zero for all values of the black hole parameters we conclude from (3.49)

$$
\eta^{+}=\alpha \xi^{+}, \quad \eta^{-}=\beta \xi^{-} \quad \alpha, \beta \in \mathbf{R}
$$

this shows that the most general Killing vector is a linear combination of $\partial / \partial t$ and $\partial / \partial \phi$ 


\section{Global Structure}

The study of global properties of the $2+1$ black hole reveals a strong coincidence with the $3+1$ case. The Penrose diagrams and maximal extensions are exactly the same as those of a $3+1$ black hole immersed in anti-de Sitter space.

\subsection{Kruskal coordinates}

We follow the analysis of [10]. For the line element

$$
d s^{2}=-\left(N^{\perp}\right)^{2} d t^{2}+\left(N^{\perp}\right)^{-2} d r^{2}+r^{2}\left(N^{\phi} d t+d \phi\right)^{2}
$$

one may introduce a Kruskal coordinate patch around each of the roots of $\left(N^{\perp}\right)^{2}=0$ to bring the metric to the form

$$
d s^{2}=\Omega^{2}\left(d u^{2}-d v^{2}\right)+r^{2}\left(N^{\phi} d t+d \phi\right)^{2}
$$

where $t=t(u, v)$.

If there is only one root $(J=0)$ then the Kruskal coordinates cover the whole space. When two roots coincide, there are no Kruskal coordinates [11].

For definiteness, we start with $r_{+}$. The Kruskal coordinates around $r_{+}$are defined by

Patch $K_{+}$:

$$
r_{-}<r \leq r_{+}\left\{\begin{aligned}
U_{+} & =\left[\left(\frac{-r+r_{+}}{r+r_{+}}\right)\left(\frac{r+r_{-}}{r-r_{-}}\right)^{r_{-} / r_{+}}\right]^{1 / 2} \sinh a_{+} t \\
V_{+} & =\left[\left(\frac{-r+r_{+}}{r+r_{+}}\right)\left(\frac{r+r_{-}}{r-r_{-}}\right)^{r_{-} / r_{+}}\right]^{1 / 2} \cosh a_{+} t \\
U_{+} & =\left[\left(\frac{r-r_{+}}{r+r_{+}}\right)\left(\frac{r+r_{-}}{r-r_{-}}\right)^{r_{-} / r_{+}}\right]^{1 / 2} \cosh a_{+} t \\
V_{+} & =\left[\left(\frac{r-r_{+}}{r+r_{+}}\right)\left(\frac{r+r_{-}}{r-r_{-}}\right)^{r_{-} / r_{+}}\right]^{1 / 2} \sinh a_{+} t
\end{aligned}\right.
$$

with

$$
a_{+}=\frac{r_{+}^{2}-r_{-}^{2}}{l^{2} r_{+}},
$$

The angular coordinate (denoted $\phi_{+}$) is chosen on $K_{+}$so that the constant of integration appearing in the solution of (2.14) is fixed to give

$$
N^{\phi}\left(r_{+}\right)=0
$$


The metric takes the form (4.2), with the conformal factor

$$
\Omega^{2}(r)=\frac{\left(r^{2}-r_{-}^{2}\right)\left(r+r_{+}\right)^{2}}{a_{+}^{2} r^{2} l^{2}}\left(\frac{r-r_{-}}{r+r_{-}}\right)^{r_{-} / r_{+}} \quad r_{-}<r<\infty .
$$

With the choice of $\phi$ leading to (4.5), the term $N^{\phi} d t$ in (4.2) remains regular at $r_{+}$.

Similarly, around $r_{-}$, one defines

Patch $K_{-}$:

$$
0<r \leq r_{-}\left\{\begin{aligned}
U_{-} & =\left[\left(\frac{-r+r_{-}}{r+r_{-}}\right)\left(\frac{r+r_{+}}{-r+r_{+}}\right)^{r_{+} / r_{-}}\right]^{1 / 2} \cosh a_{-} t \\
V_{-} \leq r \leq r_{+} & =\left[\left(\frac{-r+r_{-}}{r+r_{-}}\right)\left(\frac{r+r_{+}}{-r+r_{+}}\right)^{r_{+} / r_{-}}\right]^{1 / 2} \sinh a_{-} t \\
U_{-}= & {\left[\left(\frac{r-r_{-}}{r+r_{-}}\right)\left(\frac{r+r_{+}}{-r+r_{+}}\right)^{r_{+} / r_{-}}\right]^{1 / 2} \sinh a_{-} t } \\
V_{-}= & {\left[\left(\frac{r-r_{-}}{r+r_{-}}\right)\left(\frac{r+r_{+}}{-r+r_{+}}\right)^{r_{+} / r_{-}}\right]^{1 / 2} \cosh a_{-} t }
\end{aligned}\right.
$$

with

$$
a_{-}=\frac{r_{-}^{2}-r_{+}^{2}}{l^{2} r_{-}}
$$

This time, one chooses the angular coordinate $\phi_{-}$so that $N^{\phi}\left(r_{-}\right)=0$. The metric takes the form (4.2) with

$$
\Omega^{2}(r)=\frac{\left(r_{+}^{2}-r^{2}\right)\left(r+r_{-}\right)^{2}}{a_{-}^{2} r^{2} l^{2}}\left(\frac{r_{+}-r}{r_{+}+r}\right)^{r_{+} / r_{-}} \quad 0<r<r_{+} .
$$

The overlap of the patches $K_{+}$and $K_{-}\left(r_{-}<r<r_{+}\right)$will be called $K$. Just as in the $3+1$ case one may maximally extend the geometry by glueing together an infinite number of copies of patches $K_{+}, K_{-}$. We will not illustrate graphically that extension in terms of Kruskal coordinates, but will rather go to the more economical Penrose diagrams.

\subsection{Penrose diagrams $\left(r_{+} \neq r_{-}\right)$}

The Penrose diagrams are obtained by the usual change of coordinates

$$
U+V=\tan \left(\frac{p+q}{2}\right) \quad U-V=\tan \left(\frac{p-q}{2}\right)
$$


We define the inverse transformation by taking the usual determination of the inverse tangent, namely the one that lies between $-\pi / 2$ and $+\pi / 2$.

Consider first the case $J=0$. From (4.10) and (4.3) (with $r_{-}=0$ ) it is easy to prove that, (i) $r=\infty$ is mapped to the lines $p= \pm \frac{1}{2} \pi$, (ii) the singularity $r=0$ is mapped to the lines $q= \pm \frac{1}{2} \pi$ and (iii) the horizon is mapped to $p= \pm q$. The Kruskal and Penrose diagrams associated with this geometry are shown in Fig.3.

Next consider the case of the rotating black hole. By making the change of coordinates (4.10) in the two patches defined in Sec.(4.1) we find one Penrose diagram for each patch. These are shown in Figs. (4a, b).

The regions shown as $K$ in parts (a) and (b) of Fig.4 are to be identified because they are the overlap. Now, the original black hole coordinates covered $K$ and one region III in (4.a), and $K$ and one region $\mathbf{I}$ in (4.b). However, one wants to obtain a "maximal causal extension" (i.e., a maximal extension without closed timelike curves). To this effect one must first include the other two regions in each diagram and then glue together an infinite sequence of them, as shown in Fig.(4.c).

\subsection{Extreme cases $M=0$ and $M=|J| / l$}

\subsection{1 $M=0$}

The metric is

$$
d s^{2}=-(r / l)^{2} d t^{2}+(r / l)^{-2} d r^{2}+r^{2} d \phi^{2} .
$$

Defining the null dimensionless coordinates

$$
u=\frac{t}{l}-\frac{l}{r}, \quad v=-\frac{t}{l}-\frac{l}{r}
$$

we find

$$
d s^{2}=r^{2} d u d v+r^{2} d \phi^{2}
$$

and pass directly to Penrose coordinates by

$$
U=\tan \frac{1}{2}(p+q), \quad V=\tan \frac{1}{2}(p-q) .
$$

The relation between the radial coordinate $r$ and $p, q$ is 


$$
-r=l \frac{\cos p+\cos q}{\sin p}
$$

and the metric takes the form

$$
d s^{2}=l^{2} \frac{d p^{2}-d q^{2}}{\sin ^{2} p}+r^{2} d \phi^{2} .
$$

From (4.15) it is easy to show that the origin is mapped to the segment of the lines $p=\pi \pm q$ running from $p=0$ to $p=\pi$ while spacelike infinity is mapped to the segment of the $p=\pi$ line that closes the triangle shown in Fig.(5a).

\subsection{2 $M=|J| / l$}

The metric is

$$
d s^{2}=-\frac{\left(r^{2}-r_{+}^{2}\right)^{2}}{r^{2} l^{2}} d t^{2}+\frac{r^{2} l^{2}}{\left(r^{2}-r_{+}^{2}\right)^{2}} d r^{2}+r^{2}\left(N^{\phi} d t+d \phi\right)^{2}
$$

where $r=r_{+}=l(M / 2)^{1 / 2}$ is the horizon. Introducing the null coordinates $U=t+r^{*}$ and $V=-t+r^{*}$ where $r^{*}$ is the tortoise coordinate

$$
r^{*}=\int \frac{d r}{\left(N^{\perp}\right)^{2}}=\frac{-r l^{2}}{2\left(r^{2}-r_{+}^{2}\right)}+\frac{l^{2}}{4 r_{+}} \ln \left|\frac{r-r_{+}}{r+r_{+}}\right|
$$

and defining the Penrose coordinates $p, q$ as in (4.14) we obtain the line element

$$
d s^{2}=\frac{4\left(N^{\perp}\right)^{2} l^{2}\left(d p^{2}-d q^{2}\right)}{(\cos p+\cos q)^{2}}+r^{2}\left(N^{\phi} d t+d \phi\right)^{2} .
$$

From

$$
\frac{\sin p}{\cos q+\cos p}=\frac{-r l}{2\left(r^{2}-r_{+}^{2}\right)}+\frac{l}{4 r_{+}} \ln \left|\frac{r-r_{+}}{r+r_{+}}\right|,
$$

one sees that the lines $r=r_{+}$are at $\pm 45^{\circ}$, whereas $r=0$ is at $p=(k \pi)^{+}$and $r=\infty$ at $p=(k \pi)^{-}$. [By $p=(k \pi)^{+}$, we mean that $r \rightarrow 0$ as $p \rightarrow k \pi$ from value greater than $k \pi$, and similarly, $r \rightarrow \infty$ as $p \rightarrow k \pi$ from values smaller than $k \pi$ ]. If we take for $p$ the usual determination of the arc tangent in (4.14), so that the region $0<r<r_{+}$ is mapped on the triangle bounded by $p=0(r=0)$ and $p=q=\pi, p-q=\pi$, then we must take in the region $r>r_{+}$a different determination. Indeed, one must glue the triangle corresponding to $r>r_{+}$to the triangle corresponding to $0<r<r_{+}$ along the sides $r=r_{+}$at $45^{\circ}$, and not along the vertical sides (which are $r=\infty$ in 
the region $r>r_{+}$and $r=0$ in the region $r<r_{+}$). For instance, one could map $r>r_{+}$into the triangle bounded by $p+q=\pi, p-q=-\pi$ and $p=\pi$. Once this is done, one can go safely across $r=r_{+}$because the zero of $N^{\perp}$ in (4.19) is cancelled by the zero in the denominator. To achieve the maximal extension one then needs to include an infinite sequence of triangles as shown in Fig.(5.b) (the original black hole geometry just included two adjacent triangles).

\section{$5 \quad$ Instability of metric regularity at $r^{2}=0$. Chronology Protection}

The point of view taken in this article is that the region $r^{2}<0$ must be cut out from the spacetime because it contains closed timelike lines (see Fig.6 for a Penrose diagram that includes the forbidden region). This is a consistent point of view and leads to a close analogy with the black hole in $3+1$ dimensions. There is, however, a compelling additional argument for considering the spacetime as ending at $r=0$. It is the fact that the introduction of matter produces a curvature singularity at $r=0$. This can be easily seen in simple examples and we believe it to be a general feature (with the possible exception of very "fine-tuned" couplings). The first example is the collapse of a cloud of dust with $J=0$ [12]. One can then verify that the matter will reach infinite density at $\mathrm{r}=0$. In this case only the part of the surface $r=0$ that intersects the history of the dust becomes singular. This is due to the fact that the dust "probes" only part of the spacetime. However, in the case of a field- such as the electromagnetic field - which is our second example - all the spacetime is probed. As it was indicated in [1], the introduction of a Maxwell field that depends only on the radial coordinate yields an electromagnetic field for which the gauge invariant scalar $F_{\mu \nu} F^{\mu \nu}$ is proportional to $r^{-2}$ and thus is singular at all points on the surface $r=0$.

Therefore, in view of the curvature singularities that are brought in by matter couplings, it seems not only reasonable, but also compulsory, to exclude the region $r^{2}<0$ from the spacetime.

The collapsing dust is also interesting in that it may be regarded as a mechanism for producing, without effort, closed timelike lines from a perfectly reasonable initial condition ( with the help of a negative cosmological constant though!). However, 
one sees, first of all, that the closed timelike lines are hidden behind the horizon at $r=r_{+}>0$ (Fig. 7). But, moreover, if - say - an electromagnetic field is brought in, a barrier of infinite curvature is introduced at $r=0$. This makes the closed timelike lines not reachable from $r^{2}>0$. In this sense we see that "chronology is protected" [13] in the $2+1$ black-hole. 


\section{Acknowledgements}

Informative discussions with Steven Carlip, Frank Wilczek, and Edward Witten are gratefully acknowledged. M. B. holds a Fundación Andes Fellowship and M.H. gratefully acknowledges the hospitality of the Institute for Advanced Study where the research reported in this paper was partially carried out. This work was supported in part by grants 0862/91 and 0867/91 of FONDECYT (Chile), grant PG/082/92 of Departamento Postgrado y Postítulo, Universidad de Chile, by research funds from F.N.R.S (Belgium), by a European Communities research

contract, and by institutional support provided by SAREC (Sweden) and Empresas Copec (Chile) to the Centro de Estudios Científicos de Santiago. 


\section{Appendix A. One Parameter Subgroups of $S O(2,2)$ A.1 Description of the problem}

The purpose of this Appendix is to provide a complete classification of the inequivalent one-parameter subgroups of $S O(2,2)$. Two one-parameter subgroups $\{g(t)\}$ and $\{h(t)\}, t \in \mathbf{R}$, are said to be equivalent if and only if they are conjugate in $S O(2,2)$, i.e.,

$$
g(t)=k^{-1} h(t) k, \quad k \epsilon S O(2,2)
$$

By an $S O(2,2)$ rotation of the coordinate axes in $\mathbf{R}^{4}$, one can then map $g(t)$ on $h(t)$. Since one-parameter subgroups are obtained by exponentiating infinitesimal transformations, the task at hand amounts to classifying the elements of the Lie algebra $s o(2,2)$ up to conjugation.

Now, the elements of $s o(2,2)$ are described by antisymmetric tensors $\omega_{a b}=-\omega_{b a}$ in $\mathbf{R}^{4}$. If one conjugates the infinitesimal transformation $R^{a}{ }_{b}=\delta^{a}{ }_{b}+\varepsilon \omega^{a}{ }_{b}$ by $k \epsilon S O(2,2),\left(k^{T} \eta k=\eta, \quad \eta=\operatorname{diag}(--++)\right)$, one finds that the antisymmetric matrix $\omega \equiv\left(\omega_{a b}\right)$ transforms as

$$
\omega \rightarrow \omega^{\prime}=k^{T} \omega k, \quad k \epsilon S O(2,2)
$$

Hence we have to classify antisymmetric tensors under the equivalence relation (A.2).

\section{A.2 Strategy}

Any linear operator $M$ can be uniquely decomposed as the sum of a semi-simple (diagonalizable over the complex numbers) linear operator $S$ and a nilpotent operator $N$ that commute,

$$
\begin{gathered}
M=S+N, \\
{[S, N]=0}
\end{gathered}
$$

with

$$
N^{q}=0 \text { for some q }
$$


and

$$
S=L^{-1} \text { (diagonal matrix) } L, \text { for some } L
$$

(Jordan - Chevalley decomposition of $M$ ).

The eigenvalues of $S$ coincide with those of $M$ and provide an intrinsic characterization of $S$. When the eigenvalues of $S$ are non-degenerate, the nilpotent operator $N$ is identically zero and $M$ is thus completely characterized (up to similarity) by its eigenvalues. However, if some eigenvalues are repeated, $N$ may be non-zero and $M$ cannot be reconstructed from the knowledge of its eigenvalues: one needs also information about its nilpotent part (the dimensions of the irreducible invariant subspaces).

We shall construct the sought-for invariant classification of elements of $s o(2,2)$ by means of the Jordan - Chevalley decomposition of the operator $\omega^{a}{ }_{b}$. Since $\eta^{a b} \neq \delta_{a b}$ for $S O(2,2)$, the operator $i \omega^{a}{ }_{b}$ is, in general, not hermitian. Accordingly, it may possess a non-trivial nilpotent part when its eigenvalues are degenerate. The classification of the possible $\omega^{a}{ }_{b}$ is analogous to the invariant classification of the electromagnetic field in Minkowski space and is also reminiscent of the Petrov classification of the Weyl tensor in General Relativity.

Because the matrix $\omega_{a b}$ is real and antisymmetric, there are restrictions on its eigenvalues. These constraints are contained in the following elementary Lemmas.

Lemma 1: If $\lambda$ is an eigenvalue of $\omega_{a b}$, then $-\lambda$ is also an eigenvalue of $\omega_{a b}$.

Proof: From

$$
\left(\omega_{a b}-\lambda \eta_{a b}\right) l^{b}=0
$$

one infers the characteristic equation

$$
\operatorname{det}(\omega-\lambda \eta)=0
$$

But then $0=\operatorname{det}(\omega-\lambda \eta)^{T}=\operatorname{det}(-\omega-\lambda \eta)=\operatorname{det}(\omega+\lambda \eta)$, i.e., $-\lambda$ is also a root of the characteristic equation.

Lemma 2: If $\lambda$ is an eigenvalue, then $\lambda^{*}$ is also an eigenvalue. 
Proof: This is a consequence of the reality of $\omega_{a b}$, which implies that the characteristic equation (A.8) has real coefficients.

\section{A.2.1 Types of eigenvalues}

It follows from these theorems that the four eigenvalues of $\omega$ are of the following four possible types:

1. $\lambda, \quad-\lambda, \quad \lambda^{*}, \quad-\lambda^{*}, \quad \lambda=a+i b, \quad a \neq 0 \neq b$

2. $\lambda_{1}=\lambda_{1}^{*}, \quad-\lambda_{1}, \quad \lambda_{2}=\lambda_{2}^{*}, \quad-\lambda_{2}, \quad\left(\lambda_{1}\right.$ and $\lambda_{2}$ real $)$

3. $\lambda_{1}, \quad-\lambda_{1}=\lambda_{1}^{*}, \quad \lambda_{2}, \quad-\lambda_{2}=\lambda^{*}, \quad\left(\lambda_{1}\right.$ and $\lambda_{2}$ imaginary $)$

4. $\lambda_{1}=\lambda_{1}^{*},-\lambda_{1}, \quad \lambda_{2},-\lambda_{2}=\lambda_{2}^{*}, \quad\left(\lambda_{1}\right.$ real, $\lambda_{2}$ imaginary $)$

In each case, the eigenvalues involve only two independent real numbers, whose knowledge is equivalent to knowing the two Casimir invariants.

$$
I_{1}=\omega^{a b} \omega_{a b}, \quad I_{2}=\frac{1}{2} \epsilon^{a b c d} \omega_{a b} \omega_{c d}
$$

[If one replaces $S O(2,2)$ by $S O(4), i \omega^{a}{ }_{b}$ is hermitian and therefore diagonalizable. Hence there is no nilpotent part and $i \omega^{a}{ }_{b}$ is completely characterized by its eigenvalues and thus by $I_{1}$ and $I_{2}$.]

Multiple roots can occur only in the following circumstances:

- Cases (2) and (3), when $\lambda_{1}=\lambda_{2}$ (or $-\lambda_{2}$ ). If $\lambda_{1} \neq 0$, then $\lambda_{1}$ and $-\lambda_{1}$ are distinct roots. If $\lambda_{1}=0$, then 0 is a quadruple root; or

- Cases (2),(3) or (4), when one of the roots vanishes.

\section{A.2.2 Types of antisymmetric tensors}

For simple roots, one can give a unique canonical form to which any matrix $\omega_{a b}$ with a given set of eigenvalues can be brought to by an $S O(2,2)$ transformation. This is the form of $\omega_{a b}$ in the basis where $\omega^{a}{ }_{b}$ is diagonal. In the presence of multiple roots, there are inequivalent canonical forms because $\omega^{a}{ }_{b}$ may contain a non-trivial 
nilpotent part $N$. But for each possible type of $N$, there is a unique canonical form. These canonical forms are all derived in the next subsections.

We shall say that the matrix $\omega^{a b}$ is of type $k$ if its nilpotent part is of order $k, N^{k}=0$. The types $\mathbf{I}$ and $\mathbf{I I}$ can be further classified according to the reality properties of the roots. We thus define:

Type $\mathbf{I}(N=0)$

$\mathbf{I}_{a}: 4$ complex roots $\lambda,-\lambda, \lambda^{*},-\lambda^{*}\left(\lambda \neq \pm \lambda^{*}\right)$.

$\mathbf{I}_{b}: 4$ real roots $\lambda_{1},-\lambda_{1}, \lambda_{2},-\lambda_{2}$.

$\mathbf{I}_{c}: 4$ imaginary roots $\lambda_{1},-\lambda_{1}, \lambda_{2},-\lambda_{2}$.

$\mathbf{I}_{d}: 2$ real $\left(\lambda_{1}\right.$ and $\left.-\lambda_{1}\right)$, and two imaginary roots $\left(\lambda_{2}\right.$ and $\left.-\lambda_{2}\right)$.

Type II $\left(N \neq 0, N^{2}=0\right)$

$\mathbf{I I}_{a}: 2$ real double roots, $\lambda$ and $-\lambda$.

$\mathbf{I I}_{b}: 2$ imaginary double roots, $\lambda$ and $-\lambda$.

$\mathbf{I I}_{c}$ : 1 double root $(0)$ and 2 simple roots $(\lambda$ and $-\lambda$, with $\lambda$ real or imaginary. Type III $\left(N^{2} \neq 0, N^{3}=0\right)$ : one quadruple root, zero.

Type IV $\left(N^{3} \neq 0, N^{4}=0\right)$ : one quadruple root, zero.

We shall write in all cases

$$
\lambda=a+i b
$$

We close this section by proving the following useful Lemma.

Lemma 3: Let $v^{a}$ and $u^{a}$ be eigenvectors of $\omega^{a}{ }_{b}$ with respective eigenvalues $\lambda$ and $\mu$,

$$
\omega^{a}{ }_{b} v^{b}=\lambda v^{a}, \quad \omega^{a}{ }_{b} u^{b}=\mu u^{a} .
$$

Then $v_{a} u^{a}=0$ unless $\lambda+\mu=0$. In particular, if $\lambda \neq 0$, then $v^{a}$ is a null vector.

Proof: One has $u_{a} \omega^{a}{ }_{b} v^{b}=\lambda u_{a} v^{a}=-\mu u^{a} v_{a}$, and thus $(\lambda+\mu) u^{a} v_{a}=0$.

We now proceed to the explicit determination of the canonical forms.

\section{A.3 Type $\mathbf{I}_{a}$}

One has by definition of type $\mathbf{I}_{a}$,

$$
\omega_{a b} l^{b}=\lambda l_{a}
$$




$$
\begin{aligned}
\omega_{a b} m^{b} & =-\lambda m_{b} \\
\omega_{a b} l^{* b} & =\lambda^{*} l_{a}^{*} \\
\omega_{a b} m^{* b} & =-\lambda^{*} m_{a}^{*}
\end{aligned}
$$

where the eigenvectors $l^{a}, l^{* a}, m^{a}, m^{a *}$ are complex and linearly independent. The only scalar products that can be different from zero are $l^{a} m_{a}$ and $l^{a *} m_{a}^{*}$. They cannot vanish since the metric would then be degenerate. By scaling $m_{a}$ if necessary one can assume $l^{a} m_{a}=1$. One then has also $l^{a *} m_{a}^{*}=1$. The metric is given by

$$
\eta_{a b}=l_{a} m_{b}+l_{a}^{*} m_{b}^{*}+[a \leftrightarrow b]
$$

since

$$
\left(\eta_{a b}-l_{a} m_{b}-l_{a}^{*} m_{b}^{*}-[a \leftrightarrow b]\right) u^{b}
$$

is zero whenever $u^{a}$ equals $l^{a}, m^{a}, l^{a *}, m^{b *}$. The tensor $\omega^{a b}$ is given by

$$
\omega^{a b}=\lambda\left(l_{a} m_{b}-l_{b} m_{a}\right)+\lambda^{*}\left(l_{a}^{*} m_{b}^{*}-l_{b}^{*} m_{a}^{*}\right)
$$

because this reproduces (A.12a $)$-( A.12d $)$.

Our goal is to achieve a canonical expression for $\omega^{a}{ }_{b}$ over the real numbers. Therefore we decompose the vectors $l_{a}$ and $m_{a}$ into their real and imaginary components

$$
l_{a}=u_{a}+i v_{a}, \quad m_{a}=n_{a}+i q_{a}
$$

(the transformation $l_{a}, m_{a}, l_{a}^{*}, m_{a}^{*} \rightarrow u_{a}, v_{a}, n_{a}, q_{a}$ is invertible and so, the vectors $u_{a}, v_{a}, n_{a}$, and $q_{a}$ form a basis). This gives

$$
\begin{aligned}
\eta_{a b} & =2\left(u_{a} n_{b}-v_{a} q_{b}\right)+[a \leftrightarrow b] \\
\omega_{a b} & =2 a\left(u_{a} n_{b}-v_{a} q_{b}\right)-2 b\left(u_{a} q_{b}+v_{a} n_{b}\right)-[a \leftrightarrow b]
\end{aligned}
$$

In the orthonormal basis where the vectors $u_{a}, v_{a}, n_{a}, q_{a}$, have components $u_{a}=$ $\left(0, \frac{1}{2}, \frac{1}{2}, 0\right), \quad n_{a}=\left(0,-\frac{1}{2}, \frac{1}{2}, 0\right), \quad v_{a}=\left(\frac{1}{2}, 0,0, \frac{1}{2}\right), \quad$ and $q_{a}=\left(\frac{1}{2}, 0,0,-\frac{1}{2}\right), \omega_{a b}$ take the form 


$$
\omega_{a b}=\left(\begin{array}{rrrr}
0 & b & 0 & a \\
-b & 0 & a & 0 \\
0 & -a & 0 & b \\
-a & 0 & -b & 0
\end{array}\right)
$$

Eq. (A.18) is the canonical form of an antisymmetric tensor of type $\mathbf{I}_{a}$. The Casimir invariants are found from (A.9) to be

$$
\begin{aligned}
& \left.I_{1}=4\left(b^{2}-a^{2}\right)\right) \\
& \left.I_{2}=4\left(b^{2}+a^{2}\right)\right)
\end{aligned}
$$

\section{A.4 Type $\mathrm{I}_{b}$}

One has, by definition of type $\mathrm{I}_{b}$,

$$
\begin{gathered}
\omega_{a b} l^{b}=\lambda_{1} l_{a}, \omega_{a b} m^{b}=-\lambda_{1} m_{a} \\
\omega_{a b} n^{b}=\lambda_{2} n_{a}, \omega_{a b} u^{b}=-\lambda_{2} u_{a}
\end{gathered}
$$

The vectors $l^{a}, m^{a}, n^{a}$, and $u^{a}$ are real and linearly independent, and the nonvanishing scalar products are $l \cdot m$ and $n \cdot u$. Straightforward steps yield then, in an orthonormal basis, the canonical form

$$
\omega_{a b}=\left(\begin{array}{rrrr}
0 & 0 & 0 & -\lambda_{2} \\
0 & 0 & -\lambda_{1} & 0 \\
0 & \lambda_{1} & 0 & 0 \\
\lambda_{2} & 0 & 0 & 0
\end{array}\right)
$$

The Casimir invariants are given by 


$$
\begin{aligned}
& I_{1}=-2\left(\lambda_{1}^{2}+\lambda_{2}^{2}\right), \\
& I_{2}=4 \lambda_{1} \lambda_{2} .
\end{aligned}
$$

\section{A.5 Type $\mathbf{I}_{c}$}

One has, by definition of type $\mathbf{I}_{c}$

$$
\begin{aligned}
\omega_{a b} l^{b} & =i b_{1} l_{a}, \quad \omega_{a b} l^{b *}=-i b_{1} l_{a}^{*} \\
\omega_{a b} m^{b} & =i b_{1} m_{a}, \quad \omega_{a b} m^{b *}=-i b_{1} m_{a}^{*}
\end{aligned}
$$

The only non-vanishing scalar products are $l_{a} l^{a *}$ and $m_{a} m^{a *}$. One can rescale $l_{a}$ and $m_{a}$ so that $l \cdot l^{*}= \pm 1, m \cdot m^{*}=\mp 1$. If $l \cdot l^{*}=1$, then $m \cdot m^{*}=-1$ and vice

versa. [Through $l^{a}=\frac{1}{\sqrt{2}}\left(u^{a}+i v^{a}\right)$, one associates to a vector $l^{a}$ obeying $l_{a} l^{a *}=1$, two real vectors $u^{a}, v^{a}$, such that $u_{a} u^{a}=1=v_{a} v^{a}, u_{a} v^{a}=0$. So, if $l_{a} l^{a^{*}}=1$, one must have $m_{a} m^{a *}=-1$ in order to agree with the signature $(--++)$ of the metric.]

One obtains the final canonical form

$$
\omega_{a b}=\left(\begin{array}{rrrr}
0 & b_{1} & 0 & 0 \\
-b_{1} & 0 & 0 & 0 \\
0 & 0 & 0 & b_{2} \\
0 & 0 & -b_{2} & 0
\end{array}\right)
$$

for $\omega_{a b}$ in a real orthonormal basis.

The Casimir invariants are found to be

$$
\begin{aligned}
& I_{1}=2\left(b_{1}^{2}+b_{2}^{2}\right), \\
& I_{2}=4 b_{1} b_{2} .
\end{aligned}
$$




\section{A.6 Type $\mathbf{I}_{d}$}

Type $\mathbf{I}_{d}$ does not exist. Indeed, the real eigenvalue brings a block of signature $(+-)$, while the imaginary eigenvalue brings a block of signature $(++)$ or $(--)$. This is inconsistent with signature $(--++)$.

\section{A.7 Role of the Casimir invariants for type I}

If one compares (A.19a), A.19b), (A.22a), (A.22b) and (A.25a), (A.25b), one sees that the Casimir invariants completely characterize the matrices $\omega_{a b}$ of type I. If $\mathrm{I}_{1} \pm \mathrm{I}_{2}$ are both positive, the type is type $\mathbf{I}_{c}$. If $\mathrm{I}_{1} \pm \mathrm{I}_{2}$ are both negative, the type is type $\mathbf{I}_{b}$. Otherwise, the type is $I_{a}$. Furthermore, the eigenvalues can be reconstructed from $I_{1}$ and $I_{2}$. The roots are degenerate when $I_{1}+I_{2}$ or $I_{1}-I_{2}$ vanish. It is easy to see that $\mathrm{I}_{1} \pm \mathrm{I}_{2}$ are the Casimir invariants of the two algebras $\operatorname{so}(2,1)$ contained in $s o(2,2)=,s o(2,1) \oplus s o(2,1)$. The self-dual and anti-self-dual (real)

matrices $\omega_{a b}^{ \pm}=\omega_{a b} \pm \frac{1}{2} \epsilon_{a b}{ }^{c d} \omega_{c d}$ define irreducible representations of $s o(2,2)\left(\omega_{a b}^{+}\right.$ transforms as a vector under the first $s o(2,1)$, while $\omega_{a b}^{-}$transforms as a vector under the second.) One has $2 I_{1}=\omega_{a b}^{+} \omega^{+a b}$ and $2 I_{2}=\omega_{a b}^{-} \omega^{-a b}$. There is however, no particular advantage in working with the self-dual and anti-self-dual components of $\omega_{a b}$ in the subsequent discussion. For that reason, we shall not perform the split.

\section{A.8 Type $\mathrm{II}_{a}$}

By definition of type $\mathbf{I I}_{a}$, there are two doubly degenerate, non-zero, real eigenvalues $\lambda$ and $-\lambda$. Each eigenvalue has at least one eigenvector, thus one can find $l^{a}$ and $m^{a}$ such that

$$
\begin{aligned}
\omega_{a b} l^{b} & =\lambda l_{a} \\
\omega_{a b} m^{b} & =-\lambda m_{a}
\end{aligned}
$$

Within each invariant subspace we can introduce an additional vector to complete $l, m$ to a basis. Since $\omega^{a}{ }_{b}$ has a nilpotent part, at least one of the additional vectors will not be an eigenvector. We can thus write, without loss of generality, 


$$
\begin{aligned}
\omega_{a b} u^{b} & =\lambda u_{a}+l_{a} \\
\omega_{a b} s^{b} & =-\lambda s_{a}+\alpha m_{a}
\end{aligned}
$$

It follows from (A.26a), (A.26b) and (A.27a), (A.27b) that $l \cdot l=l \cdot m=l \cdot u=0$. Hence, since the metric is non-degenerate we must have $l \cdot s \neq 0$. This implies in turn that $\alpha$ must be different from zero since (A.27a), (A.27b) gives $l \cdot s+\alpha m \cdot u=0$. By a rescaling of $m$ we can set $\alpha=1$, so one has

$$
\omega_{a b} s^{b}=-\lambda s_{a}+m_{a}
$$

The remaining scalar products are evaluated as follows. First, one can take $u^{a} s_{a}=0$ since one can redefine $u^{a} \rightarrow u^{a}+\rho l^{a}$ without changing any of the previous relations. Second, by multiplying (A.27a) with $u^{a}$, one gets, using $u^{a} l_{a}=0$, that $u^{a} u_{a}=0$. One then finds from (A.28) $u^{a} m_{a}=-1$ as the only remaining nonvanishing scalar product.

The metric and antisymmetric tensor $\omega_{a b}$ read

$$
\begin{aligned}
\eta_{a b} & =l_{a} s_{b}-m_{a} u_{b}+[a \leftrightarrow b] \\
\omega_{a b} & =\lambda\left(l_{a} s_{b}-u_{a} m_{b}\right)-l_{a} m_{b}-[a \leftrightarrow b]
\end{aligned}
$$

In a suitable orthonormal frame, this gives

$$
\omega_{a b}=\left(\begin{array}{rrrr}
0 & 1 & 1 & \lambda \\
-1 & 0 & \lambda & 1 \\
-1 & -\lambda & 0 & 1 \\
-\lambda & -1 & -1 & 0
\end{array}\right)
$$

When $\lambda \neq 0$, a simpler, equivalent canonical form, can be achieved by replacing $m_{a}$ by $m_{a}^{\prime}+l_{a} / 2 \lambda$ and $s_{a}$ by $s_{a}^{\prime}+u_{a} / 2 \lambda$. This leaves $\eta_{a b}$ unchanged

$$
\eta_{a b}=l_{a} s_{b}^{\prime}-m_{a}^{\prime} u_{b}+[a \leftrightarrow b],
$$


but modifies $\omega_{a b}$ to

$$
\omega_{a b}=\lambda\left(l_{a} s_{b}^{\prime}-u_{a} m_{b}^{\prime}\right)+l_{a}\left(u_{b}-m_{b}^{\prime}\right)-[a \leftrightarrow b],
$$

which, in an appropriate orthonormal frame, yields

$$
\omega_{a b}=\left(\begin{array}{rrrr}
0 & 0 & 0 & \lambda \\
0 & 0 & \lambda & 1 \\
0 & -\lambda & 0 & 1 \\
-\lambda & -1 & -1 & 0
\end{array}\right)
$$

The forms (A.30) and (A.33) are not equivalent when $\lambda=0$. It is only (A.30) that is if type $\mathrm{II}_{a}$ in that case, since (A.33) with $\lambda=0$ possesses a non trivial nilpotent part of order 3 and is thus of type III. The Casimir invariants are found to be

$$
\begin{aligned}
& I_{1}=-4 \lambda^{2}, \\
& I_{2}=4 \lambda^{2} .
\end{aligned}
$$

i.e., they are exactly the same as those of (A.22a), (A.22b) with $\lambda_{1}=\lambda_{2}$. However, the canonical forms (A.30) or (A.33) are not equivalent to (A.21) with $\lambda_{1}=\lambda_{2}$ since they possess a non trivial nilpotent part, while (A.21) does not for any value of $\lambda_{1}$, $\lambda_{2}$.

\section{A.9 Types $\mathrm{II}_{b}$ and $\mathrm{II}_{c}$}

The analysis of type $\mathbf{I I}_{b}$ proceeds as for type $\mathbf{I I}_{a}$. We only quote the final canonical form in an orthonormal basis

$$
\omega_{a b}=\left(\begin{array}{cccc}
0 & b-1 & -1 & 0 \\
-b+1 & 0 & 0 & 1 \\
1 & 0 & 0 & b+1 \\
0 & -1 & -b-1 & 0
\end{array}\right)
$$


and the Casimir invariants

$$
I_{1}=4 b^{2}, \quad I_{2}=4 b^{2} .
$$

Type $\mathbf{I I}_{c}$ is incompatible with a non-degenerate metric and so it does not exist. Indeed, the equations $\omega_{a b} l^{b}=0, \omega_{a b} m^{b}=l_{a}$ ( 0 is a double root and $\omega_{b}^{a}$ is a non trivial nilpotent matrix in the corresponding invariant eigenspace), together with $\omega_{a b} u^{b}=\lambda u_{a}, \omega_{a b} v^{b}=-\lambda v_{a}$ imply $l \cdot l=l \cdot \omega \cdot m=-(l \omega) \cdot m=0, l \cdot m=\omega \cdot m=0$, $l \cdot u=\lambda^{-1} l \cdot \omega \cdot u=0, l \cdot v=-\lambda^{-1} l \cdot v=0$. So $l^{a}$ would be a non zero vector orthogonal to any vector and the metric would be degenerate.

\section{A.10 Types III and IV}

In type III, zero is a quadruple root of the characteristic equation. Since $\omega^{a}{ }_{b}$ is nilpotent of order 3 , one can find a basis such that

$$
\begin{aligned}
\omega_{a b} l^{b} & =0 \\
\omega_{a b} m^{b}=0, \quad \omega_{a b} u^{b}=m_{a}, \quad \omega_{a b} t^{b} & =u_{a} .
\end{aligned}
$$

The scalar product of $l^{a}$ with $u_{a}$ vanishes from A.37b). Similarly, $m \cdot m=$ $m \cdot u=0$. Hence $m \cdot t$ cannot vanish, say $m \cdot t= \pm 1$. Then, by a redefinition of $l^{a}, l^{a} \rightarrow l^{a}+\rho m^{a}$, one can assume $l \cdot t=0$. It follows that $l \cdot l \neq 0$ since otherwise the metric would be degenerate. We set $l \cdot l=-\varepsilon, \varepsilon= \pm 1$. By making appropriate redefinitions of $t^{a}$ if necessary and using the fact that the metric is of signature $(--++)$, one finally obtains

$$
\begin{aligned}
\eta_{a b} & =\varepsilon\left(-l_{a} l_{b}-m_{a} t_{b}-t_{b} m_{a}+u_{a} u_{b}\right) \\
\omega_{a b} & =\varepsilon\left(m_{a} u_{b}-u_{a} m_{b}\right)
\end{aligned}
$$

This yields in an appropriate orthonormal basis

$$
\text { Type } \mathbf{I I I}^{+}(\varepsilon=+1) \text {. }
$$




$$
\omega_{a b}=\left(\begin{array}{cccc}
0 & 0 & 0 & 0 \\
0 & 0 & 0 & 1 \\
0 & 0 & 0 & 1 \\
0 & -1 & -1 & 0
\end{array}\right)
$$

Type III $^{-}(\varepsilon=-1)$

$$
\omega_{a b}=\left(\begin{array}{cccc}
0 & -1 & -1 & 0 \\
1 & 0 & 0 & 0 \\
1 & 0 & 0 & 0 \\
0 & 0 & 0 & 0
\end{array}\right)
$$

The two Casimir invariants vanish for type III and yet the matrix $\omega_{a b}$ is not zero.

Type IV does not exist. Indeed for the case of nilpotency of order 4, one has $\omega_{a b} l^{b}=0, \omega_{a b} m^{b}=l_{a}, \omega_{a b} u^{b}=m_{a}$ and $\omega_{a b} t^{b}=u_{a}$. By taking the scalar product of the equation with $l^{a}$, one finds $l \cdot l=l \cdot m=l \cdot u=0$. So $l \cdot t \neq 0$, say $l \cdot t=k$. But then $u \cdot m=m \cdot \omega t=-l \cdot t \neq 0$ (from the last relations), while the equations $\omega_{a b} u^{b}=m_{a}$ and the antisymmetry of $\omega_{a b} u^{b}$ imply $u \cdot m=0$. This contradiction shows that type IV is inconsistent. 


\section{A.11 Summary of Results}

We summarize our results by giving for each type the canonical form of the Killing vector $(1 / 2) \omega^{a b} J_{a b}$ and the corresponding Casimir invariants in a table.

\begin{tabular}{|r|l|c|c|}
\hline Type & Killing vector & $\frac{1}{4} I_{1}$ & $\frac{1}{4} I_{2}$ \\
\hline $\mathbf{I}_{a}$ & $b\left(J_{01}+J_{23}\right)-a\left(J_{03}+J_{12}\right)$ & $b^{2}-a^{2}$ & $b^{2}+a^{2}$ \\
$\mathbf{I}_{b}$ & $\lambda_{1} J_{12}+\lambda_{2} J_{03}$ & $-\frac{1}{2}\left(\lambda_{1}^{2}+\lambda_{2}^{2}\right)$ & $\lambda_{1} \lambda_{2}$ \\
$\mathbf{I}_{c}$ & $b_{1} J_{01}+b_{2} J_{23}$ & $\frac{1}{2}\left(b_{1}^{2}+b_{2}^{2}\right)$ & $b_{1} b_{2}$ \\
$\mathbf{I I}_{a}$ & $\lambda\left(J_{03}+J_{12}\right)+J_{01}-J_{02}-J_{13}+J_{23}$ & $-\lambda^{2}$ & $\lambda^{2}$ \\
& or & & \\
& $\lambda\left(-J_{03}+J_{12}\right)-J_{13}+J_{23}(\lambda \neq 0)$ & $-\lambda^{2}$ & $-\lambda^{2}$ \\
$\mathbf{I I}_{b}$ & $(b-1) J_{01}+(b-1) J_{23}+J_{02}-J_{13}$ & $b^{2}$ & $b^{2}$ \\
$\mathbf{I I I}^{+}$ & $-J_{13}+J_{23}$ & 0 & 0 \\
$\mathbf{I I I}^{-}$ & $-J_{01}+J_{02}$ & 0 & 0 \\
\hline
\end{tabular}

Table 1. Classification of one-parameter subgroups of $S O(2,2)$

Note that for the second canonical form of type $\mathbf{I I}_{a}$, valid when $\lambda \neq 0$, we have replaced $J_{03}$ by $-J_{03}$ to comply with the form given in the text. This amounts to replace $\lambda_{2}$ by $-\lambda_{2}$, and can be acheived by redefinining $\xi^{0}$ as $-\xi^{0}$. This is why the second Casimir invariant, which is not parity-invariant, changes its sign.

The cases of interest for the black hole are $\mathbf{I}_{b}, \mathbf{I I}_{a}$ and $\mathbf{I I I}^{+}$, for which the eingenvalues of $\omega_{a b}$, namely $\pm r_{+} / l$ and $\pm r_{-} / l$ are all real. (These cases exist only because the signature of the metric is $(--++)$ ). Type $\mathbf{I}_{b}\left(\right.$ with $\left.\lambda_{1} \neq \lambda_{2}\right)$ describe a general black hole with $|J|<M l$, type $\mathbf{I I}_{a}$ describes an extreme black hole with non-zero mass, while type $\mathbf{I I I}^{+}$describes the ground state with $M=0$. The type becomes more and more special [from four distinct real roots to one single real root (zero)] as one goes from the general black hole to the ground state.

It is interesting to notice that if one expresses $r_{+}$and $r_{-}$as functions of $J$ and $M$ and goes beyond the extreme limit $|J|=M l$, the roots $r_{+}$and $r_{-}$become complex conjugates. This strongly suggests that type $\mathbf{I}_{a}$ describes the spacetime whose metric is obtained by setting $|J|>M l$ in the black hole line element. On the other hand, if one keeps $|J|<M l$ and takes $M<0$, the roots $r_{+}$and $r_{-}$become two different purely imaginary numbers. This strongly suggests that there is a close relationship between type $\mathbf{I}_{c}$ and the negative mass solutions of [5]. 
Finally, on an even more parenthetical note, we mention that for the Euclidean black hole the group $S O(2,2)$ is replaced by $S O(3,1)$. In that case the eigenvalues of $\omega^{a}{ }_{b}$ are of the form $(a,-a, i b,-i b)$ with real $a$ and $b$. This form may be obtained from that of type $\mathbf{I}_{b}$ above by setting $M_{E u c}=M, J_{E u c}=-i J$ in the formula (2.22), expressing the eigenvalues in terms of $M$ and $J$. This is just the prescription for the (real) Euclidean continuation of the Minkowskian signature black hole [see, for example[1].

\section{Appendix B. Smoothness of the Black Hole Geometry}

This Appendix addresses the question of whether the smoothness of anti-de Sitter space subsists after the identifications leading to the black hole are made. That is, we ask whether the quotient spaces we deal with are Hausdorff manifolds. The conclusion is that this is so when $J \neq 0$, but when $J=0$ the Hausdorff manifold structure is destroyed at $r=0$.

As discussed by Hawking and Ellis [9], the quotient spaces are Hausdorff manifolds if and only if the action of the identification subgroup $H=\{\exp 2 \pi k \xi, k \in \mathbf{Z}\}$ is properly discontinuous, namely, if the following properties hold,

(i) Each point $Q \epsilon$ adS has a neighbourhood $U$ such that $(\exp 2 \pi k \xi)(U) \cap U=\phi$ for all $k \in \mathbf{Z}, k \neq 0$; and

(ii) If $P, Q \epsilon a d S$ do not belong to the same orbit of $H$ (i.e., there is no $k \epsilon \mathbf{Z}$ such that $(\exp 2 \pi k \xi)(P)=Q)$, then there are neighborhoods $B$ and $B^{\prime}$ of $P$ and $Q$ respectively such that $(\exp 2 \pi k \xi)(B) \cap B^{\prime}=\phi$ for all $k \in \mathbf{Z}$.

To proceed with the analysis we introduce the Euclidean norm

$$
\left[\left(u^{\prime}-u\right)^{2}+\left(v^{\prime}-v\right)^{2}+\left(x^{\prime}-x\right)^{2}+\left(y^{\prime}-y\right)^{2}\right]^{1 / 2}
$$

on $\mathbf{R}^{4}$. The norm of the Killing vector

$$
\xi=\frac{r_{+}}{l}\left(u \frac{\partial}{\partial x}+x \frac{\partial}{\partial u}\right)-\frac{r_{-}}{l}\left(v \frac{\partial}{\partial y}+y \frac{\partial}{\partial v}\right)
$$


is bounded from below by $r_{-}>0$,

$$
\begin{aligned}
\|\xi \cdot \xi\|_{E} & =\left[\frac{r_{+}^{2}}{l^{2}}\left(u^{2}+x^{2}\right)+\frac{r_{-}^{2}}{l^{2}}\left(v^{2}+y^{2}\right)\right] \\
& =\left[\frac{r_{+}^{2}-r_{-}^{2}}{l^{2}}\left(u^{2}+x^{2}\right)+\frac{r_{-}^{2}}{l^{2}}\left(u^{2}+x^{2}+v^{2}+y^{2}\right)\right]^{1 / 2} \\
& \geq r_{-}>0 \quad\left(\text { on } u^{2}+v^{2}=x^{2}+y^{2}+l^{2}\right)
\end{aligned}
$$

Let $Q_{0}$ be a point of anti-de Sitter space with coordinates $\left(u_{0}, v_{0}, x_{0}, y_{0}\right)$ satisfying $u_{0}^{2}+v_{0}^{2}-x_{0}^{2}-y_{0}^{2}=l^{2}$. Its successive images $Q_{n}$ are given by

$$
\begin{aligned}
& u_{n}=(\cosh \alpha) u_{0}+(\sinh \alpha) x_{0} \\
& x_{n}=(\sinh \alpha) u_{0}+(\cosh \alpha) x_{0} \\
& v_{n}=(\cosh \beta) v_{0}-(\sinh \beta) y_{0} \\
& y_{n}=-(\sinh \beta) v_{0}+(\cosh \beta) v_{0}
\end{aligned}
$$

with $n \in \mathbf{Z}, \quad \alpha=2 \pi r_{+} / l, \quad \beta=2 \pi r_{-} / l$. The Euclidean distance $d_{E}\left(Q_{0}, Q_{n}\right),(n \neq 0)$ between $Q_{0}$ and $Q_{n}$ is bounded from below by

$$
d_{E}\left(Q_{0}, Q_{n}\right) \geq l \sqrt{2(\cosh \beta-1)}>0,(n \neq 0) .
$$

Indeed, one has

$$
\begin{aligned}
& \left(u_{n}-u_{0}\right)^{2}+\left(x_{n}-x_{0}\right)^{2}+\left(v_{n}-v_{0}\right)^{2}+\left(y_{n}-y_{0}\right)^{2} \\
& \quad \geq\left|\left(u_{n}-u_{0}\right)^{2}-\left(x_{n}-x_{0}\right)^{2}\right|+\left|\left(v_{n}-v_{0}\right)^{2}-\left(y_{n}-y_{0}\right)^{2}\right| \\
& \quad=2(\cosh n \alpha-1)\left|u_{0}^{2}-x_{0}^{2}\right|+2(\cosh n \beta-1)\left|v_{0}^{2}-y_{0}^{2}\right| \\
& \geq 2(\cosh \beta-1)\left[\left|u_{0}^{2}-x_{0}^{2}\right|+\left|v_{0}^{2}-y_{0}^{2}\right|\right] \\
& \geq 2(\cosh \beta-1)\left|u_{0}^{2}-x_{0}^{2}+v_{0}^{2}-y_{0}^{2}\right| \\
& \quad=2(\cosh \beta-1) l^{2}
\end{aligned}
$$

The bound (B.9) is uniform, i.e., it does not depend on $Q_{0}$.

Let $P_{0}$ be another point of anti-de Sitter space with coordinates $\left(\bar{u}_{0}, \bar{v}_{0}, \bar{x}_{0}, \bar{y}_{0}\right)$. It is easy to see, by using formulas analogous to (B.3) for $P_{0}$, that the distance $d_{E}\left(P_{n}, Q_{0}\right)$ between $Q_{0}$ and the images of $P_{0}$ goes to infinity as $n \rightarrow \pm \infty$. Hence, 
there is a minimum "distance of approach" of the orbit of $P_{0}$ to $Q_{0}$ (which may be zero if $Q_{0}=P_{k}$ for some $k$ ). That minimum distance of approach varies continuously if one varies $P_{0}$ continuously.

Let $U$ be the open ball centered at $Q_{0}$ with radius $r<\frac{l}{2} \sqrt{2(\cosh \beta-1)}$. The image of any point of this ball by $\exp 2 \pi k \xi,(k \neq 0)$ cannot be in $U$. Otherwise the bound (B.3) would be violated. This proves (i).

Now, turn to (ii). Let $P_{0}$ be a point that is not mapped on $Q_{0}$ by any power of $\exp 2 \pi \xi$. In the open ball $U$, there can be at most one image of $P_{0}$. If there were none, by continuity, the points sufficiently close to $P_{0}$ will have no image in $U$ and thus (ii) would hold. So let us assume that there is one image of $P_{0}$ in $U$, say $P_{n}$. Let $\tilde{B}$ be an open ball centered at $P_{n}$ and entirely contained in $U$. All the images of the points in $\tilde{B}$ lie outside $U$, i.e., $(\exp 2 \pi k \xi)(\tilde{B}) \cap U=\phi$. Let $B$ " be an open ball centered at $Q_{0}$ such that $B \cap \tilde{B}=\phi$. Then $B=(\exp -2 \pi n \xi)(\tilde{B})$ and $B$ " fulfill condition (ii).

[For simplicity we have used in this analysis the simpler form of the Killing vector only appropriate for $|J|<M l$. One can easily check that for $|J|=M l$ there are no fixed points and that all the orbits go to infinity, just as for $|J|<M l$. It then easily follows that the results for $|J|<M l$ remain valid for $|J|=M l$. The details are left to the reader]

The above argument breaks down when there is no angular momentum because the Killing vector $\xi=\frac{r_{+}}{l}\left(u \frac{\partial}{\partial x}+x \frac{\partial}{\partial u}\right)$ vanishes in that case along the line $u=x=0$, which is thus a line of fixed points. This makes the bound (B.3) empty. Furthermore, each fixed point is an accumulation point for the orbits of the points obeying $u \pm x=0$ and having the same values of $v$ and $y$. Hence, both (i) and (ii) are violated if one takes for $Q$ one of the fixed points. The action of the group is not properly discontinuous. This leads to a singularity in the manifold structure of the TaubNUT type.

[This kind of singularity has been discussed in [14]. Another example of it has been found in [15]. For an analysis see [9], where a discussion of identifications under boosts in two-dimensional Minkowski space is given. To make contact with that analysis observe that near $r=0$ one can neglect the cosmological constant. The $S O(2,2)$ group goes then over to the Poincaré group in three dimensions. The identification Killing vector (3.17) becomes then a boost plus a translation in a 
transverse direction. It is the presence, in our case, of this additional transverse direction which is responsible for the smooth behavior when $J \neq 0$ : the combination of a boost and a transverse translation does not have fixed points.] 


\section{References}

[*] e-mail: cecsnet@uchcecvm

[\#] e-mail: henneaux@ulb.ac.be

[1] M. Bañados, C. Teitelboim and J. Zanelli, Phys. Rev. Lett., 69, 1849 (1992)

[2] The black hole considered here is a solution of the standard Einstein equations with a negative cosmological constant and without matter. Its properties are in very close correspondence with its counterpart in four spacetime dimensions. Models for black holes in two spacetime dimensions can also be constructed [for an early attempt see J.D. Brown, M. Henneaux and C. Teitelboim, Phys. Rev. D33, 319 (1986)] and have been the object of considerable interest recently in connection with string theory [see E. Witten, Phys. Rev. D44 314 (1991), C-G. Callan, S.B. Giddings, and J.A. Harvey and A. Strominger, Phys. Rev. D45, 1005 (1992). The action is then not the one of Einstein's theory and it includes, in the string case, a dilaton field as an essential ingredient. A related string-inspired model in three spacetime dimensions (the "black string") has also been considered [J.H. Horne and G.T. Horowitz, Nucl. Phys. B368, 444 (1992)].

[3] The charged $2+1$ black hole was briefly discussed in [1]. It is not a spacetime of constant curvature. This makes the analysis given below, in terms of identifications in anti de Sitter space, not applicable. For an independent discussion of solutions of $2+1$ gravity coupled to electromagnetism, without a cosmological constant, see I.I. Kogan, Mod. Phys. Lett. A7, 2341 (1992).

[4] For signature $(+++)$ there is a theorem stating that any geodesically complete space of constant negative curvature is a quotient of "Euclidean anti-de Sitter" (i.e. the three-dimensional Lobachevsky plane) by a discrete subgroup of O(3,1). [W.P. Thurston, Geometry and Topology on Three-Manifolds, Princeton Lecture Notes, 1979 (unpublished)]. S. Carlip informs us that an equally clear-cut theorem does not seem to be available for signature $(-++)$ [see, in this context G. Mess, Lorentz Spacetimes of Constant Curvature, IHES/M/90/28 preprint, unpublished]. However - as persuasively argued by E. Witten (private 
communication) - it was natural to expect the black hole geometry to also be a quotient and this indeed turned out to be the case.

[5] T. Regge and C. Teitelboim, Ann. of Phys. (NY) 88, 286 (1974).

[6] J.D. Brown and M.Henneaux, Commun. Math. Phys. 104, 207 (1986)

[7] S. Deser, R. Jackiw and G.'t Hooft, Ann. Phys. (NY) 152, 220 (1984). S. Deser and R. Jackiw, Ann. Phys. (NY) 153, 405 (1984). A. Staruszkiewicz, Acta Phys. Pol. 24, 734 (1963). J. Gott and M. Alpert, Gen. Rel. 6 Grav. 16, 3 (1984).

[8] The condition for the quotient space to be smooth is that the action of the isometry group generated by $\xi$ be "properly discontinuous", see S. Hawking and G.F.R. Ellis, The Large Scale Structure of Spacetime, (Cambridge University Press, Cambridge, 1973). As discussed in Appendix B, in the black hole case this condition is met when the angular momentum is different from zero. However, when $\mathrm{J}=0$, the group action is not properly discontinuous and the singularity is of the type present in Taub-NUT space.

[9] One may regard 2+1 gravity as a Chern-Simon theory for $S O(2.2)$ [E. Witten, Nucl. Phys. B 311, 46 (1988)]. The holonomies of that approach are then $\exp 2 \pi n \xi$ with the Killing vector $\xi$ used in the identification regarded as an element of the Lie algebra of $S O(2.2)$. This correspondence appears to hold under rather general conditions on the manifold [see e.g., the work by Mess 44] and also S. Carlip, Class. and Quant. Gravity 8, 5 (1991)]. For the black geometry, the Chern-Simons holonomies have been evaluated directly by D. Cangemi, M. Leblanc and R. B. Mann (Gauge Formulation of the Spinning Black Hole in (2+1) - Dimensional Anti-de Sitter Space, MIT Preprint CTP \#2162, 1992, unpublished). Their results agree with our expressions (3.19) (applicable when $|J|<M l$ ). However, since $\xi \cdot \xi$ is a smooth surface (recall sec. 3.2.2) there is no source there for the curvature tensor (or anywhere else as a matter of fact). One gets a non-trivial holonomy -that can be thought of as a flux integralbecause of the non-trivial topology ("charge without charge". See J.A. Wheeler Geometrodynamics, Academic Press, New York 1992.) 
[10] R.H. Boyer and R.W. Lindquist, J. Math. Phys. 8, 265 (1967), B. Carter, Phys. Rev. 141, 1242, (1966); 174, 1559 (1968), J. Graves and D. Brill, Phys. Rev. 120, 1507 (1960).

[11] B. Carter, Phys. Lett. 21, 423 (1966).

[12] S.F. Ross and R.B. Mann, "Gravitationally Collapsing Dust in $(2+1)$ dimensions". Preprint WATPHYSTH 92/07, unpublished.

[13] S.W. Hawking, Phys. Rev. D. 46, 603 (1992).

[14] C.W. Misner, in Relativity Theory and Astrophysics I: Relativity and Cosmology, J. Ehlers, ed., Lectures in Applied Mathematics, 8 (American Mathematical Society), p. 160 .

[15] C. Nappi and E. Witten, Phys. Lett. 293 B, 309 (1992). 


\section{FIGURE CAPTIONS}

\section{Figure 1. Poincaré Patches}

(a) Section with surface $y=0$. The solid lines have $u+x=0, \quad y=0$. These curves are lightlike and asymptotic to $\lambda=(k+1 / 2) \pi$. The pattern is periodic in $\lambda$.

(b) Section with surface $x=0$. The solid lines (including the axis $\lambda=0$ ) have $u+x=0, x=0$ in anti-de Sitter space. The pattern is again periodic in $\lambda$. As one lets the angle $\theta$ approach $\pm \pi / 2$, the lines $u+x=0$ become more and more horizontal until they reach the configuration shown.

\section{Figure 2. Regions determined by the norm of $\xi^{\prime}$.}

(a) Section with surface $y=0$ when $r_{-} \neq 0$. The solid lines are the curves $\xi^{\prime} \cdot \xi^{\prime}=0, y=0$. They are timelike. The dotted lines are the lines $\xi^{\prime} \cdot \xi^{\prime}=r_{-}^{2}$ $\left(u^{2}-x^{2}=0\right)$, bounding regions II and III. The lines formed by dots and segments have $\xi^{\prime} \cdot \xi^{\prime}=r_{+}^{2}, y=0$.

(b) Section with surface $x=0$ when $r_{-} \neq 0$. The surface $x=0$ has $\xi^{\prime} \cdot \xi^{\prime}>0$ everywhere when $r_{-} \neq 0$. The horizontal solid lines are the lines $\xi^{\prime} \cdot \xi^{\prime}=r_{-}^{2}$, $x=0$. The lightlike lines formed by dots and segments have $\xi^{\prime} \cdot \xi^{\prime}=r_{+}^{2}$. The region $\xi^{\prime} \cdot \xi^{\prime}>0, x=0$ splits into disconnected components separated by the horizontal lines and containing two regions I and two regions II.

(c) Section with surface $y=0$ where $r_{-}=0$. The solid lines have $\xi^{\prime} \cdot \xi^{\prime}=0, y=0$. The lines formed by dots and segments have $\xi^{\prime} \cdot \xi^{\prime}=r_{+}^{2}$. The region $\xi^{\prime} \cdot \xi^{\prime}>0$ splits into disconnected components separated by the horizontal lines with each component consisting of two regions II (and two regions I, not seen in this figure since they have no intersection with $y=0$ ). Regions III have disappeared. Note that the Killing vector $\xi^{\prime}$ is now tangent to the lightlike curves $u^{2}-x^{2}=0, y=0$.

\section{Figure 3. Spacetime diagrams for $J=0$}

(a) Kruskal diagram, (b) Penrose diagram. 
Figure 4. Penrose diagrams for $J \neq 0$. (a) Patch $K_{-}$, (b) Patch $K_{+}$, (c) Complete diagram obtained by joining an infinite sequence of patches $K_{-}, K_{+}$on the overlap $K$

Figure 5. Penrose Diagrams for the extreme cases (a) $M=0=J$, (b) $M=|J / l| \neq 0$.

Figure 6. Penrose diagrams for the maximally extended non-extremal spinning black hole $(M l>|J|>0)$, showing also the regions beyond the singularity where the Killing vector $\xi$ is timelike. Regions III' are defined by $-\infty<\xi \cdot \xi<r_{-}^{2}$ and contain regions III $\left(0<\xi \cdot \xi<r_{-}^{2}\right)$. The metric in these regions is isomorphic to the metric in regions $\mathbf{I}$ but with the roles of $t$ and $\phi$ exchanged. The singularity $r=0$ in III corresponds then to the stationary surface in I. There are closed timelike curves through each point in regions III'. These closed timelike curves cross $\xi \cdot \xi=0$.

Figure 7. Penrose diagram for a collapsing body in the case $J=0$. 